\title{
Cámaras de comercio: una nueva gestión. El enfoque del Cuadro de Mando Integral en las Cámaras Españolas
}

\author{
María-José Foncubierta-Rodríguez \\ Francisca Galiana-Tonda \\ María del Mar Galiana Rubia
}

RESUMEN: Las Cámaras de Comercio, una de las organizaciones empresariales multisectoriales más extendidas en el mundo, se caracterizan por tres condiciones que las diferencian de otras agrupaciones empresariales: no tienen fines de lucro, comprenden diversos sectores dentro de un territorio específico, y son autogestionadas. En el presente trabajo, después de tratar sobre qué tipo de entidad es una Cámara, la evolución en su organización, y la utilidad de su labor para la sociedad y la economía españolas, ponemos de manifiesto la oportunidad de que éstas modifiquen su gestión introduciendo el sistema, ya bien conocido y aplicado en el sector privado, del Cuadro de Mando Integral (CMI), como instrumento de planificación estratégica. Ello supone un cambio de filosofía en unas organizaciones que son pieza clave en el desarrollo empresarial y social de los territorios. Con el CMI las Cámaras atenderán y controlarán de forma más eficiente la realización de sus funciones a través de determinadas líneas estratégicas. Líneas que no sólo velan por los resultados financieros, sino también por la contribución en la mejora de la propia organización, de su personal, de la satisfacción de las necesidades de sus clientes, o usuarios, y del entorno socioeconómico. Y lo harán a través de indicadores, para lo cual proponemos un listado de éstos, Con ellos, las Cámaras podrán contar con una guía ágil para su gestión y control, a la par que visualizarán en la sociedad de qué manera y en qué medida contribuyen a su desarrollo.

PALABRAS CLAVE: Cuadro de Mando Integral; Cámara de Comercio; Estrategia; Mapa estratégico; Indicadores de gestión en organizaciones públicas; Desarrollo territorial; Entorno socioeconómico.

CLAVES ECONLIT: L30, M00, O00, R11.

Cómo citar este artículo / How to cite this article: FONCUBIERTA-RODRÍGUEZ, M.J. \& GALIANA-TONDA, F. \& GALIANA-RUBIA, M.M. (2020): "Cámaras de comercio: una nueva gestión. El enfoque del Cuadro de Mando Integral en las Cámaras Españolas", CIRIEC-España, Revista de Economía Pública, Social y Cooperativa, 99, 273-308. DOI: 107203/CIRIEC-E.99.14602.

Correspondencia: María-José Foncubierta-Rodríguez, Universidad de Cádiz, España, Profesora Contratada Doctora en Organización de Empresas; mariajose.foncubierta@uca.es, https://orcid.org/0000-0003-32315261; Francisca Galiana-Tonda, Profesora titular IES "Torre Almirante”, francisca.galiana.edu@juntadeandalucia. es, https://orcid.org/0000-0002-6683-0678, y María del Mar Galiana Rubia, Profesora titular IES "La Inmaculada", mmgalianarubia@lainmaculadaalgeciras.com, https://orcid.org/0000-0001-8276-8475. 


\section{EXPANDED ABSTRACT}

\section{Chambers of Commerce: a new Management. The Balanced Scorecard approach for Spanish Chambers}

Chambers of Commerce are one of the most widespread multi-sector business organizations in the world. They are characterized by three main features: they are non-profit making, they bring together various sectors within a single territory (city, region, state) and they are self-managed.

In Spain, Chambers are advisory bodies to the Administration, and represent the general interest of the local business. They pay special attention to SMEs. The strategic approach to the activities of Chambers, as well as their direction and management, will affect this interest.

For approximately a decade, Public Administrations, public companies and entities and social organizations, have begun to be aware of the benefits of renouncing the traditional management through the exclusively economic data. Progressively, they have begun to apply a more comprehensive form of management, reflecting internal and external data. Of course, the economic and financial aspects are not neglected, but this is done within an overall strategic framework. To this end, the Balanced Scorecard (BSC) tool, devised by Robert Kaplan and David Norton through value chain analysis, seems to be generally accepted as an effective instrument.

There is little literature on the treatment of this instrument in organizations of corporate or public interest: professional associations, fishermen's associations, cooperatives, foundations, port administrations, universities, city councils and other local administrations, etc. But even greater is the deficiency of studies in the case of application to Chambers of Commerce.

The aim of this work is to propose a strategic map and a detailed, though not exhaustive, table of what could be the framework of indicators that would guide the management and control of the functions of a Chamber of Commerce.

After a review of the literature on the application of this instrument in entities that defend a public or corporate interest, we move on to make a portrait of what a Chamber of Commerce is, its typologies in Europe, and its characteristics in Spain. It also reflects the regulatory changes experienced in the economic and financial regime of these entities.

In this context, the question of what's the meaning of management of a Chamber of Commerce under the philosophy of the $\mathrm{CMI}$ is answered. It could be said that success in public sector companies and non-profit organizations is not based on good financial results, but on improving the usefulness 
of their activity for society (Niven 2003). Thus, the traditional architecture of the WCC must be changed by elevating the role of the mission and clients and reducing the influence of financial indicators. However, the great economic crisis experienced in recent years has meant that the public sector, or public law entities, have also had to pay special attention to obtaining, generating and using scarce financial resources.

Within the current legal framework, and given that the Chambers are public bodies that must be concerned with obtaining the funds to finance themselves, two possibilities are open for the design of the WCC: to draw it up with the characteristics of a public sector entity, or to implement it on the basis of the general model, with the financial perspective as the main objective. Based on the models of Niven (2003) and David and Lastra (2007), a proposal is made for the Chambers of Commerce.

The strategic map is designed under a specific cause and effect methodology among the four perspectives -which correspond to the four key approaches: clients and users, economic-financial, internal processes and resources and capacities-, strategies, objectives and indicators. This provides 'an excellent tool for the validation and presentation of the organization's results' (Vega \& Lluglla, 2019:9), which is 'intuitively very attractive' (Kaplan, 2003, in De Waal, 2003:1).

The strategic lines (vertical dimension) represent the means for achieving the strategic objectives set by the organization. The action plan will be based on them. These lines are: to evaluate the needs and expectations of customers and stakeholders, to evaluate and review the strategy, adjusting the organization and the distribution of functions and responsibilities, to evaluate the economic-financial management, and to improve the Chamber's Catalogue of Services.

As main results of the study, two proposals are made in the application of the CMI philosophy to the Chambers of Commerce:

1. New Process Map. It is created on the basis of the six main functions assigned to a Chamber of Commerce in Spain, which are: creation and consolidation of companies, training, internationalization, technological modernization, information and representation, and others (such as conflict mediation).

2. Scoreboard. A distinction is made between Strategic Planning, Services, Economic Evaluation and Stakeholders indicators, following the four perspectives. Indicators are established for each one of the referred functions.

- Creation and Consolidation of Companies function: 24 indicators are proposed, classified into three aspects: Companies attended, Business promotion activities and Support activities for local companies.

- Training function: 36 indicators are proposed, classified into five aspects: Training for the unemployed, Training for the employed, Income and training costs for the unemployed, Efficiency in training for the unemployed and Efficiency in training for the employed.

- Internationalization function: 26 indicators, classified into three aspects: Support activities for internationalization, Income and costs of internationalization and Efficiency in internationalization. 
- Technological Modernization function: 27 indicators, classified into four aspects: Initiatives to support the technological modernization of local companies, Training for technological modernization, Income and costs of technological modernization and Efficiency in technological modernization.

- Information and Representation function: 41 indicators, classified into six aspects: Infrastructures, Economic-Business Policy, Environment and Strategy, Participation in Public Administration, Participation in Private Entities and Income and Costs in Information and Representation.

There are basically two limitations to the job. On the one hand, although the Process Map is built on perspectives, functions, lines of action, etc., by its own conception of a graphic guide it does not include each and every one of the chamber actions and the flows between them. On the other hand, the chart of indicators is logically not exhaustive, as has been pointed out, and does not reflect all the possible activities derived from the functions of a Chamber of Commerce.

Chambers of Commerce in Spain are public-law corporations that combine public-administrative powers with the defense of the interests of the associated entities. The modification of the traditional continental model of organization of Chambers, in force since 1911, has meant an important organizational change that has affected their financial security. Chambers have been able to react to this process by increasing the services provided to their users and the control and management thereof.

The tool to be used for the management of the new context can be the BSC model, through the design and establishment of a strategic map and an adapted set of indicators, a model of efficiency demonstrated by a wide literature in business corporations, as well as, in the last years, in corporate entities and public administrations, as it has been referenced in this work. The path of the Chambers in this transformation is long, and not exempt of complexities, given the characteristics of these organizations. Nevertheless, the authors of this work consider that it is the only one, based on the new rules of the game imposed by the regulations and the environment, that will allow them to know themselves well in all the dimensions of the scope of their strategy, and to use that knowledge for their internal and external efficiency. In this evolution, the implementation of a management system based on indicators will allow them to measure the results of their management in order to make their activities profitable and to add value to their associates, betting on a continuous improvement in the development of all their processes.

The originality of this work lies in the fact that it covers an important gap in the literature, especially as far as Chambers of Commerce are concerned. It is intended to cover this gap with a double proposal of management tools for them, under the philosophy of the BSC. Not only is a strategic map provided, but also an extensive list of indicators, classified by function, and under a codification model that enables the Chamber to better manage them by computer. 
Our contribution also has a broader scope, since any 'study on the design and implementation of these innovations [the BSC model] in specific organizations allows us to contribute to strengthening the process of dissemination and adoption of such management practices' (Aparisi, Giner \& Ripoll, 2009: 207-208).

KEYWORDS: Balanced Scorecard; Chamber of Commerce; Strategy; Strategic map; Management indicators in public organizations; Territorial development; Socioeconomic environment.

\section{Introducción}

Agruparse en comunidad y organizarse ha sido la forma más eficiente de conseguir los intereses del colectivo. Cuando lo que se defienden son intereses de carácter público, las organizaciones suelen presentarse en forma de órganos de la Administración (Ministerios, Consejerías, órganos locales, internacionales, etc.) o como Administraciones de carácter corporativo público. Dentro de estas últimas quedarían encuadradas las Cámaras de Comercio (Gorordo, 2005). En este contexto, podemos decir que las Cámaras de Comercio constituyen una de las organizaciones empresariales de tipo multisectorial más extendidas en el mundo ${ }^{1}$, caracterizadas por carecer de fines lucrativos, agrupar diversos sectores dentro de su ámbito territorial específico de actuación (ciudad, comarca, región, Estado) y ser autogestionadas.

Su origen en España se remonta al Real Decreto de 9 de abril de 1886 (Blanco, 1945), por el que se crean diversas asociaciones voluntarias de comerciantes, industriales y navieros². Las Cámaras adquirieron el carácter de establecimientos públicos en 1901, a través del Real Decreto de 21 de junio ${ }^{3}$.

1.- Solo la Cámara de España reúne a 85 cámaras nacionales y 42 cámaras en países de los cinco continentes, dando servicios a 325.856 empresas (en línea: https://www.camara.es/la-camara-de-espana/la-camara-de-espana-en-cifras [visto el 09 de marzo de 2020]). En nuestro país hay 15 cámaras de 16 países europeos, que cubren a más de 7.000 empresas (en línea: http://www.camaraseuropeas.com/es/ [visto el 09 de marzo de 2020]). Eurochambres, la Asociación de Cámaras de Comercio e Industria Europeas, cuenta con 45 miembros, con una red de 1.700 cámaras, y representa a más de 20 millones de empresas en Europa, siendo PYME más del 93\% de las mismas (en línea: https://www.camara. es/acerca-de-eurochambres [visto el 09 de marzo de 2020]). En todos los países del mundo, con excepción de Nauru, Timor Oriental y Vaticano, existen cámaras de comercio (Informe "Cámaras de Comercio en Europa y en el Mundo", 2011:13).

2.- A propuesta del Ministro de Fomento, y de acuerdo con el Consejo de Ministros, he tenido a bien disponer: Artículo 1. Las asociaciones de carácter permanente que usando de su libertad constitucional funden los comerciantes, industriales, navieros y Capitanes de la Marina mercante de altura se considerarán como Cámaras oficiales de Comercio, Industria y Navegación para los efectos de este decreto... (Gaceta de Madrid, $n^{\circ}$ 102, Tomo Il, del 12 de abril de 1886, p:110).

3.- Gaceta de Madrid no 172. 
En general, las Cámaras de Comercio tienen una doble misión: prestar servicios a sus miembros y asesorar e influir en los gobiernos para que creen un entorno empresarial favorable. En los países de la Unión Europea las Cámaras ofrecen, por lo general, servicios de asesoramiento e información, así como de promoción de la internacionalización, a sus empresas miembros. La mayoría prestan, además, actividades de formación a empresas y para el empleo, y de mediación o arbitraje.

En todos ellos, las Cámaras son órganos consultivos de la Administración, y representan el interés general del tejido empresarial local, prestando especial atención a las PYME, que suponen más del $90 \%$ del total de empresas ${ }^{4}$. Por tanto, el planteamiento estratégico de las actividades de las Cámaras, así como la dirección y la gestión de las mismas, incidirán en dicho interés.

Por ello, desde hace aproximadamente una década las Administraciones Públicas, las empresas y entidades de carácter público y las de tipo social han empezado a ser conscientes de las bondades de renunciar a la tradicional gestión a través de los datos exclusivamente de tipo económico, para pasar a aplicar una forma de gestión más integral, en la que se reflejen datos internos y externos, dintorno y entorno, lo económico-financiero pero dentro de lo estratégico. Para ello, la herramienta del Cuadro de Mando Integral (CMI), Balanced Scorecard, ideado por Robert Kaplan y David Norton ${ }^{5}$ a través del análisis de la cadena de valor, parece estar aceptada generalmente como un instrumento eficaz.

Desde su creación, hace veinticinco años, la literatura se ha visto enriquecida por estudios en los que, a pesar de definir aspectos a mejorar, se confirma su utilidad en la gestión de las empresas (Vega \& Lluglla, 2019; Quesado, Aibar \& Lima, 2018; Madsen \& Stenheim, 2015; Argüello \& Quesada, 2015; Awadallah \& Allam, 2015; Vega, 2015; Nørreklit \& Mitchell, 2014; Madsen \& Stenheim, 2014; Hoque, 2014; Basuony, 2014; Abdullah et al., 2013).

No obstante, resulta escasa aún en cuanto a su aplicabilidad en organizaciones de interés público y en asociaciones de carácter corporativo. En los últimos años se ha dado un avance en este sentido. Bajo la premisa de la necesidad de una modernización de los procesos de gestión, Valdés (2017) recomienda el $\mathrm{CMI}$, e indicadores de control, para los colegios profesionales. Adaptación que realiza el Colegio Profesional de Trabajadores Sociales de Madrid en su planificación del período 2013-2016, estableciendo, además, un Mapa de Procesos. La implantación de indicadores, con el fin de que los

4.- Según datos del Directorio Central de Empresas (DIRCE) del Instituto Nacional de Estadística (INE), y bajo el criterio del número de asalariados, para 2019, el 99,82\% de empresas en España son PYME, de las cuales el 95,71\% tienen menos de 10 empleados o ninguno.

5.- Realmente, la literatura sitúa el origen del CMI en el denominado "Tableau de Bord"-creado por el alemán Jürgen H. Daum- que, aunque en un principio sólo trataba diversos ratios de tipo financiero, fue evolucionando y combinando diferentes tipos de indicadores para el control de los procesos de negocios ("Tableau de Bord: Besser als die Balanced Scorecard?" Der Controlling Berater, 7 December 2005). Kaplan y Norton presentaron su modelo en el número de eneroffebrero de 1992 de la revista Harvard Business Review, con base en un trabajo realizado para la empresa de semiconductores Analog Devices Inc. Según Aparisi -Aparisi y González-UÚbeda, IMACC-ev, Universidad de Valencia (2001), en comparación con los antecedentes, el CMI de Kaplan y Norton conlleva como aportación principal el modo como se seleccionan o se determinan los indicadores, pues la idea de utilizar indicadores, financieros o no, tiene al menos cien años, y la idea de combinarlos tiene más de cuarenta. 
resultados sean visualizados de forma inteligible y realista, es la pieza clave en el trabajo de Rea (2018) sobre un caso concreto de CMl en sindicatos.

En cuanto a las cofradías de pescadores, el compromiso con su responsabilidad social y la sostenibilidad de la actividad pesquera constituyen la visión que ha de guiar el proceso de implantación del CMI (Jiménez, 2015). Por su parte, García-Lorenzo \& Valera-Lafuente (2019) ponen el interés en las interacciones entre las cofradías, las Administraciones Públicas y otras entidades sociales del entorno. En organizaciones cooperativas también se ha llamado la atención sobre la utilidad de la aplicación del CMI (Duvauchelle \& Merchán, 2019; Portacio-Rodríguez, 2017), para subsanar la falta de cultura de control de la gestión (Pariona \& Peña, 2020) y pasar de un estilo directivo operativo y cortoplacista a otro a largo plazo (Alzate, 2007), así como para ser más eficientes en el proceso de innovación y adaptación al entorno (Martínez-Vilanova \& Rodenes-Adam, 2009). El éxito, no obstante, ha sido relativo, en el sentido de que los autores detectan limitaciones, como que no existe consenso de indicadores propios a aplicar (Zachow \& Bertolini, 2019).

Para las fundaciones, la literatura recoge algunos estudios que, sin tratar directamente el CMI, indican el uso de indicadores para medir la eficiencia de sus actuaciones, analizando si dicha eficiencia depende de determinados factores, como su carácter público o privado, su tamaño, la composición de su patrimonio, la función del fundador, etc. (González \& Rúa, 2007), o para elaborar un Balance Social, que transmita a la sociedad el grado de cumplimiento de sus objetivos fundacionales (Cabra de Luna, 2001).

En el contexto de las Administraciones Públicas, también encontramos organismos que tienen un carácter corporativo, o de defensa de los intereses de una comunidad. Es el caso de la Administración Portuaria. Las autoridades portuarias son organismos públicos, cuya actuación, salvo en el ejercicio de sus funciones de poder público, se sujeta al ordenamiento jurídico privado. Desde la reforma reguladora de $1992^{6}$, las autoridades portuarias tienen autonomía económico-financiera y responden de las actividades que al cliente brinda el conjunto de su comunidad portuaria. La competitividad entre puertos y entre cadenas logísticas ha sido creciente, y se requiere una estrategia para gestionar la comunidad portuaria (Estrada, 2007). De ahí que se comience a plantear la utilización del CMI (Aparisi, Giner y Ripoll, 2009), a la par que el organismo estatal de Puertos del Estado las controla a través de un listado de indicadores comunes (Crespo, Giner y Ripoll, 2010, en Foncubierta, 2010:118). Esta propuesta ya era recogida por la Asociación Española de Contabilidad y Administración (AECA) en su documento "La Contabilidad de Gestión en el Sistema Portuario Español" (AECA, 2006). 
En general, también es aceptado que para la gestión más eficiente de una organización de carácter público se requiere la obtención de información útil y ágil, y que a tal fin el CMl y un sistema de indicadores pueden ser instrumentos muy válidos (Jiang \& Liu, 2014). A esta misma conclusión llegan Guimarães, Simões \& Marques (2010) cuando centran sus estudios en la progresiva reducción de recursos financieros de los que disponen las administraciones públicas. En este contexto, la eficiencia en la gestión se convierte en un requisito imprescindible. Siguiendo el proceso de un $\mathrm{CMI}$ se pueden reducir dificultades que afrontan frecuentemente las organizaciones de carácter público: falta de una organización diáfana de responsabilidades, duplicación de competencias e información imperfecta para el control y evaluación del desempeño (Lu, 2009) de cara a medir el cumplimiento de objetivos, entendidos como servicios valorados por la comunidad (Hasanah \& Novita, 2019).

Las universidades han sido, igualmente, objeto de estudio en la implantación de CMI (Ramírez, 2011; Binden, Mziu \& Suhaimi, 2014; Elola, Tejedor \& Tejedor, 2016; Al-Hayaly \& Alnajjar, 2016; Fijalkowska \& Oliveira, 2018; Kuchta et al., 2019).

También lo han sido otros organismos gubernamentales, locales o de ámbito superior (en la Administración en general: Jiang \& Liu, op cit:; Bernhard Smandek, Winkler \& Ulbig, 2010; Yetano, 2005; para ayuntamientos y Administración local: Jiang \& Liu, op cit.; Junta de Andalucía, Dirección General de Administración Local, 2004; Lizcano, 2002; en Administración sanitaria: Ribeiro, Vasconcelos y Rocha, 2019; Khiew, Chen, Shia \& Pan, 2017; Huang, Lai \& Lin, 2011; González, López-Valeiras y Gómez, 2011; en Administración deportiva: López Hernández, 2010; Viñas, 2009; López Hernández, López Villegla y Rodríguez Nieto, 2008; Navarro, 2008; Marín y García, 2007).

Sin embargo, la deficiencia en estudios de su uso en el caso concreto de las Cámaras de Comercio es notable, la literatura al respecto es muy escasa aún. Curiosamente, las Cámaras de Comercio en España han estado realizando y apoyando la formación en aplicación de esta herramienta para las empresas de su demarcación territorial, sin aplicarla a su propia organización.

Nuestro objetivo en este trabajo es el de proponer un $\mathrm{CMI}$, un mapa estratégico y un cuadro detallado, aunque no exhaustivo, de lo que podría ser el marco de indicadores que guiara, con relativa facilidad y agilidad, a la gestión y el control de las funciones camerales. Nuestra aportación, además, tiene un mayor alcance, pues todo "estudio sobre el diseño e implantación de estas innovaciones [modelo CMI] en organizaciones concretas permite contribuir a potenciar el proceso de divulgación y adopción de tales prácticas de gestión" (Aparisi, Giner \& Ripoll, op cit: 207-208). 


\section{Los modelos de Cámaras de Comercio en la Unión Europea y sus implicaciones en la organización de las mismas}

Las labores camerales pueden ser desarrolladas desde distintos enfoques organizativos. El tipo de modelo de organización empresarial que elija un país dejará una perdurable marca en su dirección política y económica.

\section{Tabla 1. Diferencias de los modelos de Cámaras de Comercio en Europa}

\begin{tabular}{|l|l|l|}
\hline \multirow{2}{*}{} & \multicolumn{2}{|c|}{ MODELOS DE CÁMARAS DE COMERCIO } \\
\cline { 2 - 3 } & Francés (continental) & Anglosajón \\
\hline Creación & Mediante ley & Asociación privada \\
\hline empriación de las & $\begin{array}{l}\text { Obligatoria para todas las empresas de la } \\
\text { demarcación de la Cámara }\end{array}$ & $\begin{array}{l}\text { Voluntaria: representación de los intereses de } \\
\text { sus miembros }\end{array}$ \\
\hline Representación & $\begin{array}{l}\text { Representación, promoción y defensa } \\
\text { del interés general de las empresas y de } \\
\text { la economía de la demarcación ante las } \\
\text { Administraciones Públicas }\end{array}$ & $\begin{array}{l}\text { Defienden exclusivamente los intereses de } \\
\text { sus asociados, frente a las administraciones } \\
\text { públicas y frente a terceros. }\end{array}$ \\
\hline Cuota cameral & Obligatoria & No existe cuota obligatoria \\
\hline Financiación & Base financiera amplia y segura & Seguridad financiera limitada \\
\hline $\begin{array}{l}\text { Finalidad de los } \\
\text { servicios }\end{array}$ & $\begin{array}{l}\text { Mayores servicios a coste mínimo para el } \\
\text { colectivo representado }\end{array}$ & $\begin{array}{l}\text { Creación de lobby } \\
\text { Rentabilización de los servicios }\end{array}$ \\
\hline $\begin{array}{l}\text { Relación con el } \\
\text { Gobierno }\end{array}$ & $\begin{array}{l}\text { Delegación de funciones que corresponden } \\
\text { al sector público: organismos oficialmente } \\
\text { independientes, pero con funciones } \\
\text { asignadas por ley }\end{array}$ & $\begin{array}{l}\text { No realizan funciones públicas: independencia } \\
\text { del Estado }\end{array}$ \\
\hline $\begin{array}{l}\text { Países europeos que } \\
\text { siguen el modelo }\end{array}$ & $\begin{array}{l}\text { Alemania, Austria, Francia, Grecia, Italia, } \\
\text { Luxemburgo, Países Bajos, Croacia y } \\
\text { Turquía. }\end{array}$ & $\begin{array}{l}\text { Reino Unido, Irlanda, Portugal, Malta, } \\
\text { Finlandia, Suecia, Dinamarca, Bélgica, Polonia, } \\
\text { República Checa, Eslovaquia, Eslovenia, } \\
\text { Estonia, Letonia, Lituania, Chipre, Bulgaria y } \\
\text { Rumanía. Islandia, Noruega y Suiza }\end{array}$ \\
\hline
\end{tabular}

NOTA: En fondo sombreado se muestran las características de las Cámaras españolas tras la Ley 4/2014.

FUENTE: Elaboración propia. 
Tradicionalmente, las Cámaras de Comercio en Europa han seguido dos modelos: el modelo francés y el modelo anglosajón (Tabla 1). Se podría añadir a los anteriores un tercer modelo, que, al combinar características de ambos, recibe el nombre de modelo mixto. No obstante, y siguiendo el informe Cámaras de Comercio en Europa y en el Mundo, realizado en 2011 para el Consejo Superior de Cámaras de Comercio de España, sea cual sea el modelo que las rige, todas las Cámaras europeas tienen en común:

- Que realizan funciones de carácter público (por ejemplo, en la emisión de documentación oficial para la exportación).

- Que cuentan con financiación pública para el desarrollo de las mismas, ya sea mediante contratos, tributos, subvenciones, etc.

- Que la financiación recibida se condiciona al nivel y calidad de los servicios públicos prestados.

- Que determinados servicios especializados de interés general, tales como la formación profesional, la asistencia en la creación de empresas, o los viveros empresariales, tan solo se llevarán a cabo si las Cámaras han recibido financiación pública para los mismos, puesto que sus costes no pueden ser sufragados por precios de mercado.

Por su parte, la legislación europea, al tener en consideración los dos modelos de Cámaras de Comercio que funcionan en los Estados miembros, no ha establecido modelo concreto de configuración jurídica ni forma de financiación.

\subsection{Las Cámaras de Comercio en España: evolución hacia un modelo "sui generis"}

Desde la aprobación en 1911 de la Ley de Bases, de 29 de junio, se ha seguido un modelo continental de organización de Cámaras, caracterizado por su adscripción forzosa y pago obligatorio de cuotas. No obstante, su configuración ha supuesto sentencias dispares y contradictorias entre el Tribunal Supremo y el Tribunal Constitucional, así como posiciones muy encontradas en el seno de este. Como consecuencia, el Real Decreto Ley 13/2010, de 3 de diciembre, de actuaciones en el ámbito fiscal, laboral y liberalizadoras para fomentar la inversión y creación de empleo, vino a romper este modelo y estableció la voluntariedad de la adscripción y consiguiente pago de cuotas ${ }^{7}$. En el apartado III de su Preámbulo se refleja que:

... la voluntariedad de la pertenencia a las Cámaras debe ser un incentivo para que cumplan sus funciones con mayor eficiencia que hasta el momento. La necesidad de asegurar el interés de las empresas por seguir contribuyendo servirá de estímulo para impulsar su modernización y consolidación como prestadoras de servicios de mayor utilidad para sus asociados (BOE núm. 293, de 3 de diciembre de 2010. Sec. I, p:101055).

7.- Según se justifica en el apartado III de su Introducción, el nuevo entorno en que se encontraban inmersas las empresas, las dificultades financieras del sector productivo y la incertidumbre sobre la fortaleza y ritmo de recuperación, implicaban la eliminación de ciertas cargas económicas directas sobre las mismas. 
Por otro lado, la voluntariedad de la pertenencia a las Cámaras debe ser un incentivo para que cumplan sus funciones con mayor eficiencia que hasta el momento. La necesidad de asegurar el interés de las empresas por seguir contribuyendo servirá de estímulo para impulsar su modernización y consolidación como prestadoras de servicios de mayor utilidad para sus asociados.

Hasta la aprobación de esta nueva norma, las Cámaras de Comercio se habían venido financiando a través de dos tipos fundamentales de recursos: el recurso cameral permanente ${ }^{8}$, con carácter obligatorio, para todos los considerados electores, y los recursos no permanentes, derivados, fundamentalmente, de la prestación de servicios a empresas y del ejercicio de sus actividades.

Este importante proceso de cambio normativo dio lugar a la Ley de Cámaras de Comercio Españolas, Ley 4/2014, de 1 de abril, Básica de las Cámaras Oficiales de Comercio, Industria, Servicios y Navegación, que las sitúa en una categoría "sui generis", a camino entre los modelos antes citados. Con ella se recupera la adscripción obligatoria de todas las empresas a las Cámaras de Comercio, pero sin obligación alguna de contribución económica a las mismas, sólo voluntaria.

\section{Tabla 2. Funciones de una Cámara de Comercio en España}

\begin{tabular}{|l|l|}
\hline FUNCIÓN & CONTENIDO \\
\hline Creación y consolidación de empresas & $\begin{array}{l}\text { Asesoramiento en la tramitación de creación de nuevas empresas, } \\
\text { especialmente en la ayuda a la PYME }\end{array}$ \\
\hline Formación y Empleo & $\begin{array}{l}\text { Formación empresarial en general. Destacan las actividades formativas en } \\
\text { materia de comercio exterior y para el empleo }\end{array}$ \\
\hline Internacionalización & Apoyo a las actividades de comercio exterior \\
\hline Modernización tecnológica & $\begin{array}{l}\text { Asistir a las empresas en la innovación en la gestión y la incorporación de } \\
\text { nuevas tecnologías }\end{array}$ \\
\hline Información y representación & $\begin{array}{l}\text { Suministrar información a las empresas y a la Administración. Participación } \\
\text { en multitud de organismos públicos }\end{array}$ \\
\hline Otras & Funciones arbitrales y competencias sobre la actividad comercial y el turismo \\
\hline
\end{tabular}

FUENTE: Elaboración propia.

8. - El Recurso Cameral Permanente había estado constituido por las siguientes exacciones: a) del $2 \%$ sobre cada una de las cuotas municipales, provinciales o nacionales del Impuesto sobre Actividades Económicas (IAE); b) del 1,5\%0 sobre los rendimientos netos de las actividades empresariales y algunas de las profesionales declarados en el Impuesto sobre la Renta de las Personas Físicas. Se practicaron liquidaciones a las personas físicas (empresarios individuales) que estaban sujetos; $y$ c) del 0,75\% sobre la cuota líquida positiva del Impuesto de Sociedades en el tramo comprendido entre 0,01 y 60.101,21€ de cuota. Para cuotas liquidas superiores se establecía una escala decreciente. Se practicaron liquidaciones a las personas jurídicas que estaban sujetas. 
El objetivo de la nueva ley ha sido el de dotar a estas instituciones de un marco legal moderno y flexible que atienda a sus necesidades actuales y refuerce su papel en el apoyo a la creación de empresas y su internacionalización. Para ello se mantiene su naturaleza de "corporaciones de derecho público" y la tutela administrativa de la Comunidad Autónoma. Permanece como finalidades principales las de representación, promoción y defensa de los intereses generales del comercio, la industria, la navegación, así como la prestación de servicios a las empresas. Las cinco contribuciones fundamentales de las Cámaras quedan resumidas en la Tabla 2.

En el terreno de lo financiero, la Ley no contempla la recuperación del recurso cameral permanente, por tanto, la financiación de las Cámaras se basará en los ingresos que se generen por los servicios que presten y por las aportaciones voluntarias de empresas, de igual modo que estableció el Real Decreto Ley 13/2010, sin olvidar los fondos europeos que pudiesen obtener. Por otra parte, las empresas que contribuyan a la financiación de las Cámaras podrán contar con una mayor presencia en sus órganos de gobierno. Como novedad es de mención que las cuotas voluntarias de empresas se destinarán preferentemente a financiar el Plan de Internacionalización.

Por su parte, la Cámara de España, como órgano de representación y coordinación de las Cámaras españolas, en sustitución del Consejo Superior desde 2014, tiene como misión principal la de representar al conjunto de las Cámaras ante instancias estatales e internacionales; así como impulsar y coordinar aquellas actuaciones que sean comunes a todas ellas. Colabora con el Ministerio para el fomento de la competitividad de las empresas, a través de Planes de Competitividad bianuales, y de Internacionalización, anuales. La Cámara de España se convierte, pues, en una entidad de referencia en la reflexión estratégica para las empresas españolas?.

9.- A este fin contribuyen, concretamente, las comisiones y comités sectoriales y transversales que puede crear en su seno. Actualmente, son doce las comisiones activadas: Formación, Universidad-Empresa, Comercio, Turismo, Industria, Energía, Digitalización, Pymes, Economía Circular, Comunicación, de Puertos y la de Internacionalización. Que la Cámara de España se crea para el interés general del tejido empresarial del país lo pone de manifiesto la frase con la que su actual Presidente nos recibía en su portal en la red, www.camara.es (visitado el 08 de agosto de 2018): Debemos fortalecer nuestras empresas para el progreso de España y el bienestar de los españoles (José Luis Bonet Ferrer). 


\section{La gestión en las Cámaras de Comercio a través de la filosofía del Cuadro de Mando Integral}

Desde un punto de vista práctico, el resultado final de la actividad de una organización proviene de la forma en que esta elige e implementa su estrategia. La estrategia ha de ser sólidamente formulada y eficazmente implantada, de forma que responda a la pregunta: ¿dónde queremos que esté nuestra entidad en un futuro, en qué posición frente a la competencia? Pero tratando únicamente datos de tipo financiero la organización se ve incapaz de saber si está cumpliendo su misión o si se halla en la senda para conseguir los retos estratégicos que se ha marcado. Su fijación ha de realizarse, en consecuencia, de forma mucho más global o integrada, a través de las siguientes tareas: valorar los objetivos de la empresa desde la perspectiva de los shareholders -accionistas- y de los stakeholders o grupos con intereses en la empresa, plasmando las exigencias de ambos colectivos en sus objetivos estratégicos (Kaplan, Norton \& Rugelsjoen, 2010), y trasladar los objetivos generales de maximización de los beneficios y de incremento del valor a objetivos operativos medibles (Cokins, 2010; Miranda \& Dalla, 2006), a alcanzar por los diferentes niveles directivos de la empresa -su compromiso es clave en el proceso (Hristov \& Chirico, 2016; Daniel, Myers \& Dixon, 2008; Assiri, Zairi \& Eid, 2006; Santos \& Fidalgo, 2005)-, y desde ellos, a todos los puestos y unidades de la entidad, enlazándolos y controlándolos con enfoque sistémico (Kaplan \& Norton, 2008).

En esta concepción de gestión integral los resultados también han de ser entendidos como sistémicos, pues los aciertos serán confirmaciones de la metodología empleada, mientras que los errores se entenderán como oportunidad para el aprendizaje. De esta manera, el sistema creado actuará, con referentes internos y externos, retroalimentándose continuamente, lo cual es capacidad propia de los sistemas abiertos. La clave de todo este proceso reside en relacionar los objetivos de resultados con variables sobre las que los diferentes directivos y trabajadores tengan competencias y, por consiguiente, puedan ejercer algún control (López Viñegla, 2003, 2004; Chavan, 2009), e ir revisando el sistema continuamente, pues no sólo se están persiguiendo fines a largo plazo, sino que la propia planificación estratégica puede ir cambiando durante su ejecución a lo largo del tiempo, dando lugar en la práctica a tres estrategias: la deliberada, la emergente, y la realmente realizada (tal y como ya señalaba Mintzberg en 1994).

Las Cámaras de España son entidades "sin ánimo de lucro, pero también sin ánimo de pérdida" (Alzate, op cit.: 187). Con la intención de adaptarse al nuevo marco jurídico, rentabilizar la prestación de servicios, y con el afán de mejora continua, han iniciado un proceso de modificación de su estructura organizativa. El proceso, aún en marcha, persigue, entre otras cuestiones, promover un significativo cambio al sustituir una gestión tradicional basada en indicadores financieros, por una gestión basada en la metodología integral, como la del CMI, con indicadores de servicios prestados. 
El modelo $\mathrm{CMl}$ se centra en responder a cuatro grandes cuestiones, y, por tanto, persigue conocer los resultados desde cuatro enfoques claves: ¿cómo se atiende a la sociedad?, perspectiva económica-financiera (financial); ¿cómo nos ven los usuarios de nuestros servicios? o perspectiva del cliente (customer); ¿en qué debemos destacar?, visión de las capacidades distintas, que es la denominada perspectiva interna (internal business); y, por último, ¿podemos continuar mejorando y creando valor?, proceso para la entropía negativa, o perspectiva de la innovación y del aprendizaje, de la entidad y de su personal (learning and growth).

No obstante, se podría afirmar que en las empresas del Sector Público y entidades sin ánimo de lucro el éxito no se basa en los buenos resultados financieros, sino en mejorar la utilidad de su actividad para la sociedad (Niven 2003). De este modo, la tradicional arquitectura del CMI debe cambiarse elevando el papel de la misión y los clientes y reduciendo la influencia de los indicadores financieros. Sin embargo, la gran crisis económica vivida en los últimos años ha provocado que también el Sector Público, o las entidades de Derecho Público, tengan que prestar especial atención a la obtención, la generación y el uso de los escasos recursos financieros.

En el marco jurídico actual, y dado que las Cámaras son organismos públicos que deben preocuparse por conseguir los fondos para financiarse, se abren dos posibilidades de diseño del CMI: elaborarlo con las características propias de una entidad del Sector Público, o implantarlo en base al modelo general, con la perspectiva financiera como objetivo principal. Niven (op.cit.) define para el Sector Público un modelo que, unido al confeccionado por David y Lastra (2007), nos permite presentar una propuesta cameral (Figura 1).

Como puede observarse, la estrategia sigue estando en el centro del sistema, aunque en algunos casos pudieran existir dificultades para establecerla de forma clara y concisa. Los objetivos de estrategia deben partir de perspectivas de mayor nivel -la misión y la visión de la entidad-, y trazar una línea de acción a largo plazo desde la situación real a la deseada, en cumplimiento de éstas.

Si una Cámara coloca la misión en la parte más alta de la estructura establecería una clara distinción con el modelo utilizado para el sector privado, ya que la atención se centraría en los clientes y en servir sus necesidades, no en alcanzar el éxito financiero. Sin embargo, esto les llevaría a una situación paradójica, pues si la Cámara no consigue la financiación necesaria para la consecución de sus objetivos, peligraría su propia supervivencia. 


\section{Figura 1. Cuadro de Mando Integral para una Cámara de Comercio en España}

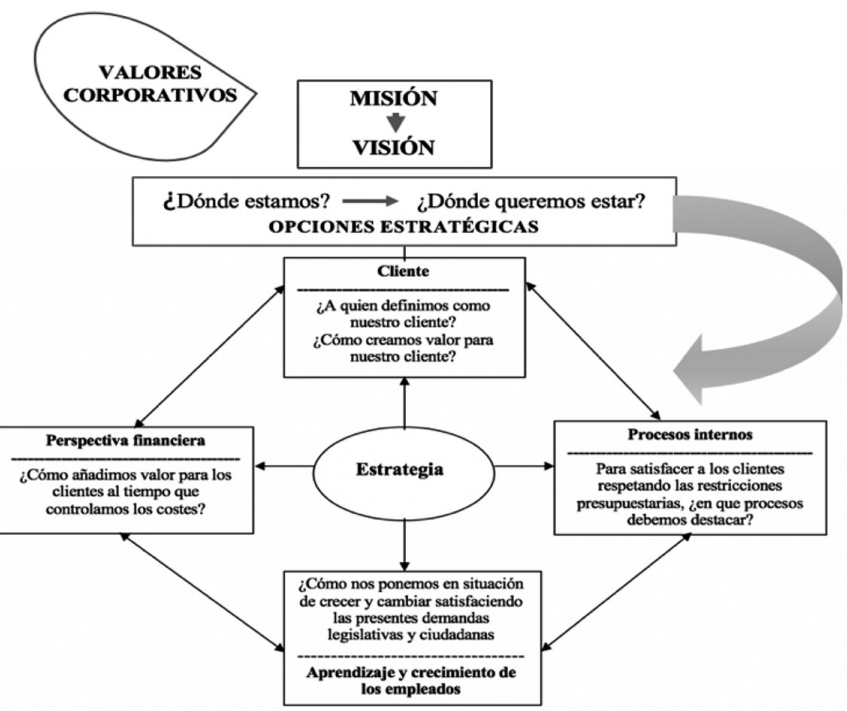

FUENTE: Elaboración propia a partir de Niven (op cit.: 380) y David y Lastra (op cit.:49).

\subsection{Mapa estratégico}

Ahora bien, ¿quién es el cliente de una Cámara? Esta cuestión, compleja ya de por sí para cualquier organismo perteneciente al Sector Público, se complica más aún en el caso de las Cámaras de Comercio. ¿Consideramos que los clientes deben ser únicamente los empresarios que voluntariamente hayan decidido realizar aportaciones económicas a la Cámara de su demarcación territorial? o ¿los clientes van a ser todas las empresas que forman parte del censo público que deben elaborar las Cámaras en su demarcación? Nos decantamos por esta última opción. 


\section{Figura 2. Perspectivas en el Mapa Estratégico de una Cámara de Comercio}
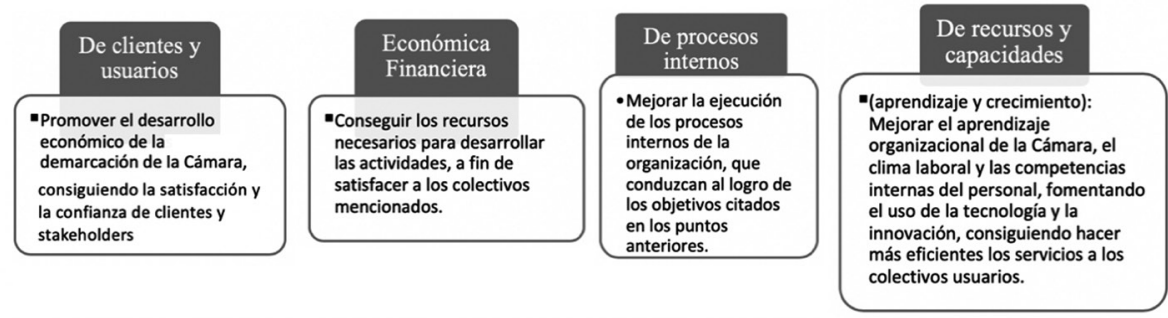

FUENTE: Elaboración propia.

Conjuntamente, las líneas estratégicas (dimensión vertical) representan el medio para la consecución de los objetivos estratégicos marcados por la organización; sobre ellas, se elaborará el plan de acciones. En el análisis vertical del mapa estratégico de una Cámara se han determinado cuatro Líneas Estratégicas básicas, que deben inspirar su actuación de cara a conseguir la misión definida Figura 3).

\section{Figura 3. Líneas estratégicas en el Mapa Estratégico de una Cámara de Comercio}

\begin{tabular}{|c|c|}
\hline $\begin{array}{c}\text { Evaluar necesidades y } \\
\text { expectativas de clientes y } \\
\text { grupos de interés } \\
\text { (stakeholders) }\end{array}$ & $\begin{array}{c}\text { Mediante la obtención de información socioeconómica, conocimiento } \\
\text { del marco coyuntural, conexión continua con clientes, y sus agrupaciones, y } \\
\text { demás grupos stakeholders, y obtención de feedback con ellos. }\end{array}$ \\
\hline $\begin{array}{c}\text { Evaluar y revisar la } \\
\text { estrategia, ajustando la } \\
\text { organización y la } \\
\text { distribución de funciones y } \\
\text { responsabilidades }\end{array}$ & $\begin{array}{c}\text { Traducción de la estrategia a los procesos internos, que procure que las } \\
\text { actividades consideradas clave sean realizadas por personal permanente y con } \\
\text { procesos estables, minimizando la dependencia de estos de coyunturas políticas } \\
\text { del entorno o de cambios en la composición de los órganos de gobierno. }\end{array}$ \\
\hline $\begin{array}{c}\text { Evaluación de la gestión } \\
\text { económica y financiera }\end{array}$ & $\begin{array}{c}\text { Obtención de recusos financieros. Elaboración de presupuestos } \\
\text { económicos en colaboración con el resto de Responsables, asegurando que } \\
\text { derivan de la estrategia y planes que la desarrollan, y que cumplen los } \\
\text { requisitos legales establecidos para las Cámaras. Seguimiento e informes } \\
\text { mensuales y liquidación anual. }\end{array}$ \\
\hline $\begin{array}{c}\text { Mejorar el Catálogo de } \\
\text { Servicios de la Cámara }\end{array}$ & $\begin{array}{c}\text { A partir del conocimiento actualizado de las necesidades de los clientes y } \\
\text { stakeholders, mediante servicios diferenciados e innovadores ofertados en base al } \\
\text { conocimiento del tejido empresarial territorial y de la capacidad de penetración. }\end{array}$ \\
\hline
\end{tabular}

FUENTE: Elaboración propia.

Paralelamente, las Cámaras han analizado sus fortalezas y debilidades, detectando, a través de éstas últimas, las principales deficiencias en su gestión, cuales son:

- Ausencia de una estrategia clara y consensuada, y de una planificación sistemática que incluya la definición de objetivos de mejora y la toma de decisiones en base a datos sobre ellos. 
- Disfuncionalidades en la organización y la responsabilidad sobre las tareas: actividades consideradas claves que se realizan por personal sin la cualificación que estas requieren, y actividades no asignadas.

- Escasa utilidad de parte de ciertos recursos, destinados a servicios poco diferenciados, a actividades que inciden sobre pocos clientes, y a programas que pueden no responder a servicios clave de la Cámara o a necesidades constatadas del mercado.

- Dependencia de criterios no estratégicos a la hora de gestionar proyectos, y confusión de tareas técnicas e institucionales.

Considerando esta situación, y ante los cambios normativos experimentados, las Cámaras han optado por comenzar el replanteamiento en las formas de su gestión a través del estudio detallado de las funciones que desarrollan (Tabla 2), y de los procesos que se ponen en marcha para la ejecución de estas, así como las interrelaciones entre ellos y con las actividades o procesos desempeñados desde y/o hacia el entorno. La toma de conciencia sobre la importancia de un planteamiento estratégico consensuado es el punto de partida a tal fin.

\section{Figura 4. Nuevo Mapa de Procesos en una Cámara de Comercio}

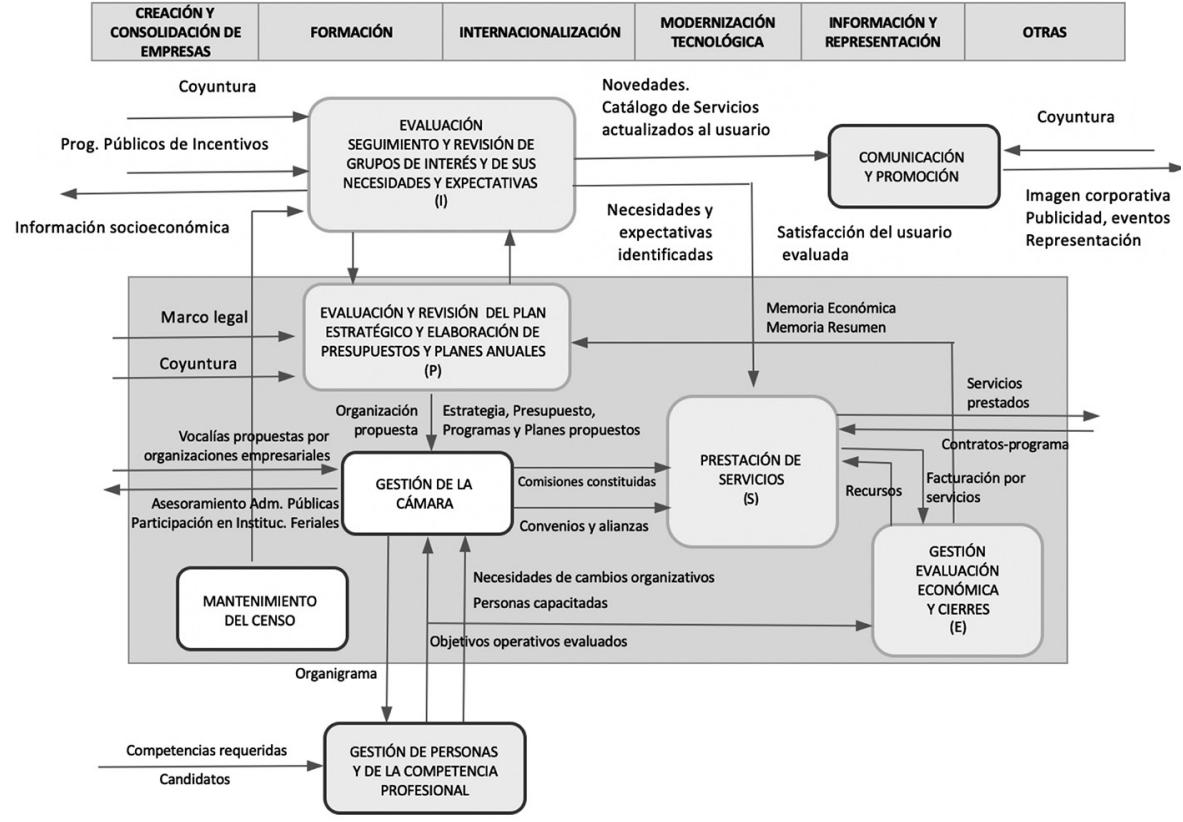

FUENTE: Elaboración propia a partir de información de la Cámara de Comercio, Industria y Navegación del Campo de Gibraltar. 
Para asistir en este proceso se ha diseñado en este trabajo lo que sería el mapa teórico al que ha de tender la entidad en cuanto a tareas a realizar, conexiones entre ellas, y relaciones con agentes externos. La Figura 4 muestra el resultado, con una clara interrelación del dintorno y el entorno de la Cámara.

\subsection{Cuadro de Indicadores}

Definido el nuevo mapa de procesos, el paso siguiente consiste en determinar los indicadores de gestión oportunos que permitan medir el grado de adecuación a los objetivos establecidos. Un indicador puede ser definido como una unidad de medida que permite el seguimiento y evaluación periódica de las variables clave de una organización, mediante su comparación en el tiempo con los correspondientes referentes externos e internos (AECA 2001, 2002). Una variable clave de negocio será aquel aspecto 0 característica que se considera crítica para el éxito de la organización a largo plazo, permitiendo a esta aumentar y/o mantener su ventaja competitiva. Los indicadores han de definirse para cada servicio o función que realice la entidad, en los aspectos de estas que sean, por tanto, más representativos o que mejor caracterice sus factores vitales (González \& Cañadas, 2008:232).

Dichos factores han de reunir, principalmente, las siguientes condiciones: ser capaz de explicar el éxito o fracaso de la organización; ser suficientemente significativo su impacto en la cuenta de resultados; ser representativo de los cambios del entorno; que origine acciones inmediatas cuando se produzca un cambio en el factor y que pueda ser mensurable o cuantificable, bien de forma directa 0 indirecta (AECA, 2002, op.cit.).

Hay que tener presente que en toda Cámara española los recursos, a partir de ahora, se van a componer fundamentalmente de aportaciones voluntarias de sus socios y de contraprestaciones por la gestión de programas y servicios públicos de apoyo a empresas, financiados por fondos provenientes de las distintas administraciones -autonómica, nacional y europea-, además de ingresos recibidos por la prestación de servicios directos y personalizados a las empresas, que podríamos denominar de carácter "privado". Por ello, ahora más que nunca, la eficacia de los servicios prestados y la adecuada administración de los recursos disponibles deben valorarse por la satisfacción de las necesidades de las empresas y stakeholders de su demarcación y el grado de cumplimiento de sus objetivos, más que por cumplimiento estricto de su presupuesto. Por tanto, es necesario determinar unos indicadores que permitan realizar un seguimiento y evaluación periódica de la actuación de la Cámara, ya que el objetivo último es analizar la gestión desarrollada con el fin de poder aplicar los correctivos necesarios para su mejora (Ospina, 2000). Los indicadores nos aportarán datos necesarios a tal efecto, pues "lo que no se define no se puede medir. Lo que no se mide, no se puede mejorar. Lo que no se mejora, se degrada siempre"10. 
En este sentido, se han de evitar dos errores potenciales: el de establecer indicadores que le resulte a la entidad más fáciles de medir en lugar de aquellos que sean realmente útiles (Cokins, op cit.), y el de excederse en el establecimiento de los mismos, gestionando información irrelevante (Santos y Fidalgo, op cit.). Para ello es necesario que el conjunto de indicadores elegidos comprenda las diferentes dimensiones del mapa de procesos, con base en las funciones, como se comentó.

\subsubsection{Una propuesta de Cuadro de Indicadores para una Cámara de Comercio en España}

Siguiendo lo indicado en el apartado III del preámbulo del Real Decreto Ley 13/2010, hemos integrado las cinco funciones principales de una Cámara de Comercio, tal y como fueron referidas en la Tabla 2, con las líneas básicas de procesos establecidos en el mapa de la Figura 4, de cara a seleccionar una serie de elementos o variables clave, $y$, a través de ello, hemos realizado una propuesta de Cuadro de Indicadores para una Cámara ${ }^{11}$. De esta forma, la información manejada sería más detallada, clara y concreta, y permitiría, por tanto, un mejor control de cada una de ellas, y de sus subactividades (Tablas 4, 5, 6, 7 y 8 ).

Por supuesto, no pretende ser exhaustivo, sino un ejemplo de cómo podría estructurarse el cuadro. Somos conscientes de que en él tienen cabida más indicadores para cada una de las funciones, y que prácticamente cada indicador podría, a su vez, ser diferenciado por diversas variables. Así, por ejemplo, en el caso de los indicadores sobre empresas, se podría diferenciar por sector de actividad de la empresa, tamaño, municipio al que pertenece, etc. La formación puede diferenciarse por contenidos, por tipología de destinatarios y de las empresas de las que forman parte, en su caso. Los referidos a encuentros, foros y otros mecanismos de participación de la Cámara podrían dividirse por tipologías, temáticas, alcance nacional o internacional, etc. Por su parte ingresos y costes pueden, y deben, ser desglosados por sus orígenes concretos. Cada Cámara deberá ajustarlo a sus propias necesidades, tanto de apartados o dimensiones a considerar dentro de cada una de las cinco funciones principales de las Cámaras, como de las variables a incluir como indicadores en cada una de ellas.

Por otro lado, el proceso debería estar informatizado a través del uso de algún software. A tal fin, y aunque existen múltiples formatos reconocidos internacionalmente en el mercado (Roncancio, 2018), los expertos destacan el predominio de un diseño interno, ad hoc, con la asistencia de asesores (Vega \& Lluglla, op cit.).

10.- Frase cuya autoría es atribuida a William Thomson Kelvin, físico y matemático británico (Lord Kelvin, 1824-907), aunque mencionada reiteradamente en la literatura socioeconómica por otros autores.

11.- Por referirse las funciones al valor que la Cámara aporta a otras entidades o personas, no están incluidas en dicha propuesta las líneas estratégicas que aparecen en el mapa estratégico (Figura 4) y que hacen relación a la gestión interna cameral, tanto en lo organizativo como en lo que respecta a aumento de su capital humano; las cuales, sin duda, deben disponer de sus indicadores para seguimiento y control. Respecto a los indicadores de procesos internos, Niven (op cit.) señala que deberían derivarse de la proposición de valor reflejada en la perspectiva del cliente. Ahora más que nunca, las Cámaras deben ofrecer un excelente funcionamiento, que con una fuerte visión les permita ser líderes en la prestación de los servicios ofertados. 
En la propuesta se han codificado los indicadores para permitir que la Cámara obtenga de manera rápida, a través de ese software, datos agregados según diferentes factores. De esta forma, puede obtenerse:

- Datos agregados de cada línea estratégica: Planificación Estratégica (P), Servicios (S), Evaluación Económica (E) o Grupos de Interés (I) (Figura 4).

- Para cada línea estratégica, su intervención en cada una de las cinco funciones principales según Tabla 2 (P.1XX; P.2.XX; P.3.XX, ...).

- Combinaciones múltiples, según las perspectivas 0 dimensiones horizontales del mapa estratégico: de tipo financiero, clientes atendidos, procesos llevados a cabo, etc.

\section{Tabla 3. Cuadro de definición de un indicador ${ }^{12}$}

(Satisfacción de clientes con el servicio X)

\begin{tabular}{|c|c|c|}
\hline \multirow{9}{*}{ 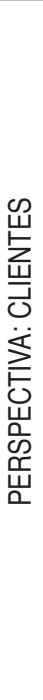 } & ÁREA DE RESULTADOS & PRESTACIÓN DE SERVICIOS \\
\hline & Nombre del indicador & Satisfacción del cliente con el servicio $\left(^{*}\right)$ \\
\hline & Descripción del indicador & $\begin{array}{l}\text { Medición del nivel de satisfacción del cliente con el servicio concreto, en } \\
\text { términos de calidad, tiempo empleado, oportunidad, información ofrecida, } \\
\text { veracidad, utilidad, etc. }\end{array}$ \\
\hline & Objetivos del indicador & $\begin{array}{l}\text { Localizar oportunidades de mejoras, a través de la detección de errores } 0 \\
\text { deficiencias referidas }\end{array}$ \\
\hline & Responsable & A designar por la entidad \\
\hline & Frecuencia de medición & Trimestral \\
\hline & Sistema de recogida de datos & $\begin{array}{l}\text { Cuestionario con Escala Likert (p.e. Del } 1 \text { al } 5 \text {, siendo 1= "Mínima } \\
\text { satisfacción" y 5="Máxima satisfacción") }\end{array}$ \\
\hline & Sistema de cálculo & Media, y valores extremos, sobre todo inferiores \\
\hline & Valores aceptables del indicador & $\begin{array}{l}\text { Valores deseables }>4,50 \\
\text { Valores aceptables: } 4,50-4,01 \\
\text { Valor mínimo aceptable: } 4,00 \\
\text { Valores que detectan deficiencias: } 3,99-3,00 \\
\text { Valores extremos: }<3,00\end{array}$ \\
\hline
\end{tabular}

(*) Se realizaría un cuadro de seguimiento similar por cada servicio prestado.

FUENTE: Elaboración propia a partir de Serna (2003).

12.- Los ítems señalados se definen como (Serna, 2003): nombre o denominación: característica, hecho o evento que se desea controlar; descripción: definición de tal característica, hecho o evento, interpretación del valor o resultado; objetivo o finalidad: fin que se persigue con el control de tal característica, hecho o evento; persona de la entidad responsable de su seguimiento; frecuencia de medición, o periodicidad con la que se medirá el indicador, para medir su evolución; sistema de recogida de la información: fuentes concretas de recopilación de datos, ya sean secundarias o primarias, y responsables de su obtención y valores aceptables: para marcar la diferencia con aquellos no aceptables, que reflejarán defectos a subsanar. 
Los indicadores vienen expresados tanto en valores relativos, porcentajes 0 ratios, como en valores absolutos, en función de la variable a medir. Para cualquiera de ellos resulta tan relevante el valor del propio indicador en un momento concreto como, sobre todo, el seguimiento de su evolución, por lo que se debería establecer una periodicidad adecuada en la medición de cada uno de ellos. A su vez, para cada uno de los indicadores se debería determinar un cuadro de definición de indicador, con todos los elementos comentados, tal y como se perfila, en la Tabla 3, con el ejemplo: "Satisfacción de la empresa con el servicio X".

\section{Reflexiones finales}

Las Cámaras de Comercio son creadas por el Estado como organizaciones de índole profesional económica, siendo corporaciones de Derecho Público que conjugan las competencias públicoadministrativas, que les corresponden como tales, con la defensa de los intereses de las entidades asociadas. La modificación del tradicional modelo continental de organización de Cámaras, vigente desde 1911, ha supuesto un importante cambio organizativo que ha afectado, a la par, a su seguridad financiera; fortaleza característica, hasta ahora, de este tipo de organizaciones. Las Cámaras han sabido reaccionar ante este proceso incrementando los servicios prestados a sus usuarios y el control y gestión de los mismos.

La herramienta utilizada para ello puede ser el modelo $\mathrm{CMI}$, a través del diseño y establecimiento de un mapa estratégico y un conjunto adaptado de indicadores, modelo de eficacia demostrada por una amplia literatura en corporaciones empresariales, así como, en los últimos años, en entidades de carácter corporativo y administraciones públicas, como se ha referenciado en este trabajo. En esta evolución, implantar un sistema de gestión basado en indicadores les permitirá medir el resultado de su gestión en pro de rentabilizar sus actividades y de añadir valor a sus asociados, apostando por una mejora continua en el desarrollo de todos sus procesos.

Además, su diseño implica que dichos indicadores surjan de la previa definición de objetivos y áreas estratégicas de actuación, esto es, obliga a la Cámara a clarificar su estrategia. El propio cambio en la gestión, al seguir el CMI, supone ya, implícitamente, una intensa actividad de mejora de procesos internos, y sería objeto de indicadores operativos (de avance de lo existente e inclusión de nuevos) que permitan medir su evolución. El binomio, satisfacción de clientes y stakeholders, y supervivencia financiera, requiere un gran esfuerzo y una filosofía permanente de optimización de los mismos. 
La nueva norma que regula las funciones de las Cámaras estableció otros servicios a realizar por parte de estas, como son los de mediación y arbitraje, que podrían, igualmente, estar reflejados en él. A nuestro entender, estos podrían considerarse como indicadores de innovación, al menos en la primera fase de implantación, para reflejarse, posteriormente, como un servicio más a evaluar, complementando los indicadores de servicios a usuarios.

Por último, ningún indicador es independiente de otro. El funcionamiento de la entidad como sistema supone que ninguno de sus componentes quede excluido de afectación, en mayor o menor grado, por lo que suceda en cualquier otro. Siempre existirá una interacción o influencia entre los indicadores que se miden, y que debería ser, a su vez, medida o analizada.

El camino de las Cámaras en esta transformación es largo, y no exento de complejidades, dadas las características de estas organizaciones. No obstante, consideramos que es el único, en base a las nuevas reglas de juego impuestas por la normativa y por el entorno, que les permitirá conocerse bien en todas las dimensiones del alcance de su estrategia, y usar ese conocimiento para su eficiencia interna y externa. 


\section{Tabla 4. Cuadro de Indicadores para la función de Creación y Consolidación de Empresas, según procesos del Mapa Estratégico}

\begin{tabular}{|c|c|c|c|c|c|c|}
\hline $\begin{array}{l}\text { FUNCIÓN A } \\
\text { EVALUAR }\end{array}$ & $\begin{array}{l}\text { INDICADORES } \\
\text { PLANIFICACIÓN ESTRATÉGICA (Código P) } \\
\text { SERVICIOS (Código S) } \\
\text { EVALUACIÓN ECONÓMICA (Código E) } \\
\text { GRUPOS INTERÉS (Código I) }\end{array}$ & $\begin{array}{l}\text { UNIDAD/ } \\
\text { PUESTO } \\
\text { RESPON- } \\
\text { SABLE } \\
\text { MEDICIÓN }\end{array}$ & $\begin{array}{l}\text { UNIDAD/ } \\
\text { PUESTO } \\
\text { RESPON- } \\
\text { SABLE } \\
\text { GESTIÓN }\end{array}$ & $\begin{array}{l}\text { PLAZO } \\
\text { O } \\
\text { PERÍO- } \\
\text { DO }\end{array}$ & $\begin{array}{l}\text { MEDIDA } \\
\text { ESTÁN- } \\
\text { DAR O } \\
\text { DESEADA }\end{array}$ & $\begin{array}{c}\text { DESVIA- } \\
\text { CIÓN EN } \\
\text { MEDIDA } \\
\text { REAL }\end{array}$ \\
\hline & $\begin{array}{l}\text { 1. CREACIÓN Y CONSOLIDACIÓN DE } \\
\text { EMPRESAS }\end{array}$ & & & & & \\
\hline $\begin{array}{c}\text { AT. EM- } \\
\text { PRESAS } \\
\text { ATENDI- } \\
\text { DAS }\end{array}$ & $\begin{array}{l}\text { S.1.AT.1. } N^{\circ} \text { de nuevas empresas tramitadas } \\
\text { S.1.AT.2. Variación anual en el } n^{\circ} \text { de nuevas } \\
\text { empresas tramitadas } \\
\text { S.1.AT.3. N }{ }^{\circ} \text { de nuevas PYME tramitadas } \\
\text { S.1.AT.4. Variación anual en el } n^{\circ} \text { de nuevas } \\
\text { PYME tramitadas } \\
\text { S.1.AT.5. } N^{\circ} \text { de nuevas PYME tramitadas } / \mathrm{n}^{\circ} \\
\text { nuevas empresas tramitadas } \\
\text { I.1.AT.1. Grado de satisfacción de las } \\
\text { empresas asociadas con esta labor } \\
\text { E.1.AT.1. Ingresos/Costes de actividad } \\
\text { tramitación } \\
\text { E.1.AT.2. Variación anual del ratio Ingreso/ } \\
\text { Costes Tramitación } \\
\text { P.1.AT.1. Presupuesto destinado a tramitación } \\
\text { nuevas empresas } \\
\text { P.1.AT.2. Variación presupuestaria anual } \\
\text { Tramitación nuevas empresas }\end{array}$ & & & & & \\
\hline $\begin{array}{l}\text { PR. ACTI- } \\
\text { VIDADES } \\
\text { DE PRO- } \\
\text { MOCIÓN } \\
\text { EMPRESA- } \\
\quad \text { RIAL }\end{array}$ & $\begin{array}{l}\text { S.1.PR.1. } N^{\circ} \text { de actividades para promoción } \\
\text { de empresas } \\
\text { S.1.PR.2. Variación anual del } n^{\circ} \text { actividades } \\
\text { para promoción empresas } \\
\text { E.1.PR.1. Ingresos/Costes de actividad } \\
\text { promoción } \\
\text { E.1.PR.2. Variación anual del ratio Ingreso/ } \\
\text { Costes Promoción } \\
\text { P.1.PR.1. Presupuesto destinado a activida- } \\
\text { des promoción } \\
\text { P.1.PR.2. Variación presupuestaria anual } \\
\text { Promoción } \\
\text { I.1.PR.1. Grado de satisfacción de las empre- } \\
\text { sas asociadas con esta labor }\end{array}$ & & & & & \\
\hline $\begin{array}{l}\text { AP. ACTI- } \\
\text { VIDADES } \\
\text { APOYO } \\
\text { EMPRE- } \\
\text { SAS LOCA- } \\
\quad \text { LES }\end{array}$ & $\begin{array}{l}\text { S.1.AP.1. } N^{\circ} \text { de iniciativas locales para apoyo } \\
\text { de empresas } \\
\text { S.1.AP.2. Variación anual del } n^{\circ} \text { de iniciativas } \\
\text { locales para apoyo a empresas } \\
\text { E.1.AP.1. Ingresos/Costes de actividad apoyo } \\
\text { E.1.AP.2. Variación anual del ratio Ingreso/ } \\
\text { Costes Apoyo } \\
\text { P.1.AP.1. Presupuesto destinado a iniciativas } \\
\text { locales apoyo } \\
\text { P.1.AP.2. Variación presupuestaria anual } \\
\text { Iniciativas locales apoyo } \\
\text { I.1.AP.1. Grado de satisfacción de las } \\
\text { empresas asociadas con esta labor }\end{array}$ & & & & & \\
\hline
\end{tabular}

FUENTE: Elaboración propia.

CIRIEC-España, Revista de Economía Pública, Social y Cooperativa 
Tabla 5. Cuadro de Indicadores para la función de Formación, según procesos del Mapa Estratégico

\begin{tabular}{|c|c|c|c|c|c|c|}
\hline $\begin{array}{l}\text { FUNCIÓN A } \\
\text { EVALUAR }\end{array}$ & INDICADORES & $\begin{array}{l}\text { UNIDAD/ } \\
\text { PUESTO } \\
\text { RESPON- } \\
\text { SABLE } \\
\text { MEDICIÓN }\end{array}$ & $\begin{array}{l}\text { UNIDAD/ } \\
\text { PUESTO } \\
\text { RESPON- } \\
\text { SABLE } \\
\text { GESTIÓN }\end{array}$ & $\begin{array}{l}\text { PLAZO } \\
\text { O } \\
\text { PERÍO- } \\
\text { DO }\end{array}$ & $\begin{array}{c}\text { MEDIDA } \\
\text { ESTÁN- } \\
\text { DAR }\end{array}$ & $\begin{array}{c}\text { DESVIA- } \\
\text { CIÓN EN } \\
\text { MEDIDA } \\
\text { REAL }\end{array}$ \\
\hline & 2. FORMACIÓN & & & & & \\
\hline $\begin{array}{c}\text { FD. FOR } \\
\text { MACIÓN } \\
\text { PARA } \\
\text { DESEM- } \\
\text { PLEADOS }\end{array}$ & $\begin{array}{l}\text { I.2.FD.1. } \mathrm{N}^{\circ} \text { de actividades para diagnosis } \\
\text { necesidades formativas desempleados } \\
\text { I.2.FD.2. Variación anual ( } \mathrm{u} \text { otro período) } \mathrm{n}^{\circ} \\
\text { actividades diagnosis necesidades formativas } \\
\text { desempleados } \\
\text { S.2.FD.1. } \mathrm{N}^{\circ} \text { de actividades formativas para } \\
\text { desempleados } \\
\text { S.2.FD.2. Variación anual del } \mathrm{n}^{\circ} \text { de activida- } \\
\text { des formativas para desempleados } \\
\text { S.2.FD.3. } \mathrm{N}^{\circ} \text { de participantes formandos } \\
\text { desempleados } \\
\text { S.2.FD.4. Variación anual del } \mathrm{n}^{\circ} \text { de formandos } \\
\text { desempleados } \\
\text { S.2.FD.5. N }{ }^{\circ} \text { de horas de formación para } \\
\text { desempleados } \\
\text { S.2.FD.6. Variación anual de horas formación } \\
\text { desempleados } \\
\text { S.2.FD.7. Contenidos priorizados en forma- } \\
\text { ción desempleados } \\
\text { I.2.FD.3. Grado de satisfacción de los forman- } \\
\text { dos desempleados }\end{array}$ & & & & & \\
\hline $\begin{array}{l}\text { FO. FOR- } \\
\text { MACIÓN } \\
\text { PARA } \\
\text { OCUPA- } \\
\text { DOS }\end{array}$ & $\begin{array}{l}\text { I.2.FO.1. } N^{\circ} \text { de actividades para diagnosis } \\
\text { necesidades formativas ocupados } \\
\text { I.2.FO.2. Variación anual (u otro período) } n^{\circ} \\
\text { actividades diagnosis necesidades formativas } \\
\text { desempleados } \\
\text { S.2.FO.1. } \mathrm{N}^{\circ} \text { de actividades formativas para } \\
\text { ocupados } \\
\text { S.2.FO.2. Variación anual del } \mathrm{n}^{\circ} \text { de activida- } \\
\text { des formativas para ocupados } \\
\text { S.2.FO.3. } \mathrm{N}^{\circ} \text { de participantes formandos } \\
\text { ocupados } \\
\text { S.2.FO.4. Variación anual del } \mathrm{n}^{\circ} \text { de formandos } \\
\text { ocupados } \\
\text { S.2.FO.5. No de horas de formación para } \\
\text { ocupados } \\
\text { S.2.FO.6. Variación anual de horas formación } \\
\text { ocupados } \\
\text { S.2.FO.7. Contenidos priorizados para forma- } \\
\text { ción ocupados } \\
\text { S.2.FO.8. Contenidos priorizados para forma- } \\
\text { ción ocupados en PYME } \\
\text { I.2.FO.3. Grado de satisfacción de los forman- } \\
\text { dos ocupados } \\
\text { I.2.FO.4. Grado de satisfacción de las empre- } \\
\text { sas a las que pertenecen los ocupados }\end{array}$ & & & & & \\
\hline
\end{tabular}




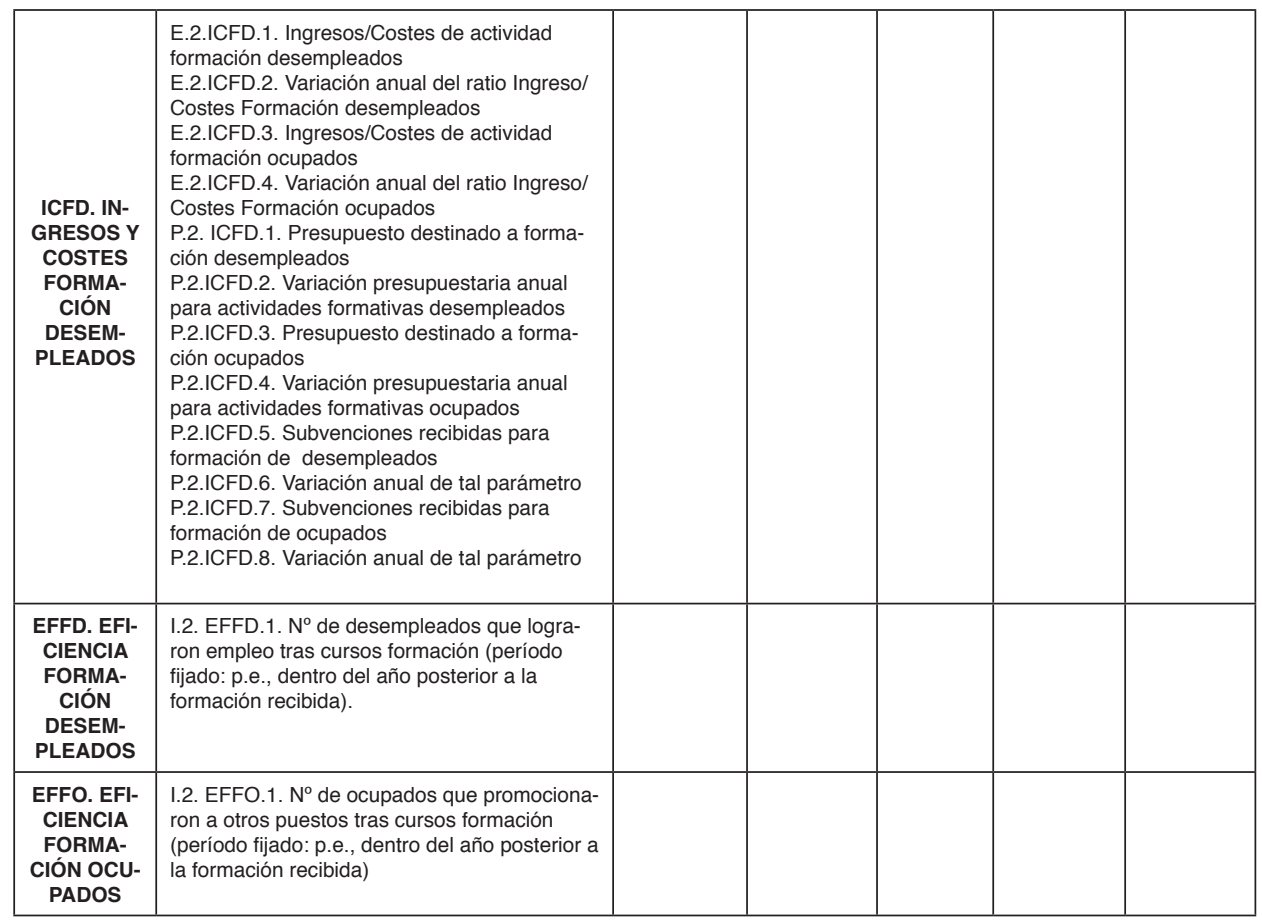

FUENTE: Elaboración propia. 
Tabla 6. Cuadro de Indicadores para la función de Internacionalización, según procesos del Mapa Estratégico

\begin{tabular}{|c|c|c|c|c|c|c|}
\hline $\begin{array}{c}\text { FUNCIÓN A } \\
\text { EVALUAR }\end{array}$ & INDICADORES & $\begin{array}{l}\text { UNIDAD/ } \\
\text { PUESTO } \\
\text { RESPON- } \\
\text { SABLE } \\
\text { MEDICIÓN }\end{array}$ & $\begin{array}{l}\text { UNIDAD/ } \\
\text { PUESTO } \\
\text { RESPON- } \\
\text { SABLE } \\
\text { GESTIÓN }\end{array}$ & $\begin{array}{l}\text { PLA- } \\
\text { ZO O } \\
\text { PERIOO- } \\
\text { DO }\end{array}$ & $\begin{array}{l}\text { MEDIDA } \\
\text { ESTÁN- } \\
\text { DAR O } \\
\text { DESEADA }\end{array}$ & $\begin{array}{c}\text { DESVIA- } \\
\text { CIÓN EN } \\
\text { MEDIDA } \\
\text { REAL }\end{array}$ \\
\hline & 3. INTERNACIONALIZACIÓN & & & & & \\
\hline $\begin{array}{c}\text { APINT. } \\
\text { ACTIVIDA- } \\
\text { DES } \\
\text { APOYO A LA } \\
\text { INTERNA- } \\
\text { CIONALIZA- } \\
\text { CIÓN }\end{array}$ & 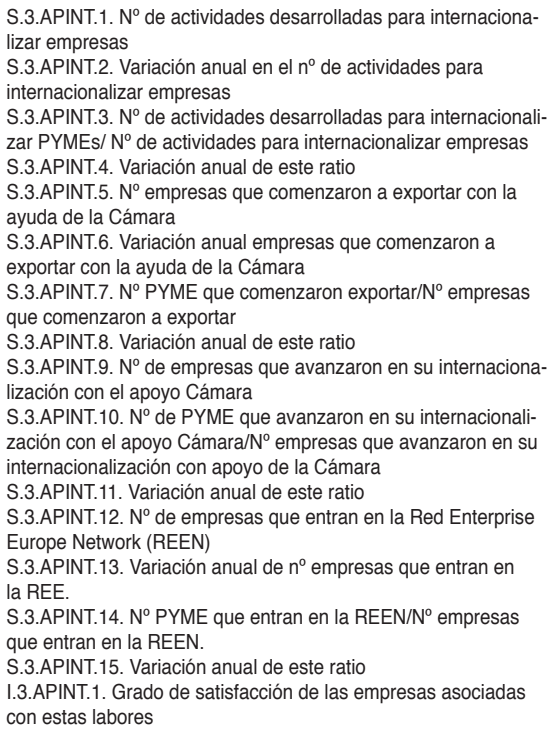 & & & & & \\
\hline $\begin{array}{l}\text { ICINT. } \\
\text { INGRESOS Y } \\
\text { COSTES DE } \\
\text { INTERNA- } \\
\text { CIONALIZA- } \\
\text { CIÓN }\end{array}$ & $\begin{array}{l}\text { E.3.ICINT.1. Ingresos/Costes actividades internacionalización } \\
\text { (general) } \\
\text { E.3.ICINT.2. Ingresos/Costes actividades inicio exportación } \\
\text { E.3.ICINT.3. Ingresos/Costes actividades avance en internacio- } \\
\text { nalización } \\
\text { E.3.ICINT.4. Variación anual de estos ratios } \\
\text { P.3.ICINT.1. Presupuesto destinado a actividades de internacio- } \\
\text { nalización (general) } \\
\text { P.3.ICINT.2. Presupuesto destinado a actividades de inicio } \\
\text { exportación } \\
\text { P.3.ICINT.3. Presupuesto destinado a actividades avance } \\
\text { internacionalización } \\
\text { P.3.ICINT.4. Variación presupuestaria anual para estas activi- } \\
\text { dades }\end{array}$ & & & & & \\
\hline $\begin{array}{l}\text { EFINT. EFI- } \\
\text { CIENCIA EN } \\
\text { INTERNA- } \\
\text { CIONALIZA- } \\
\text { CIÓN }\end{array}$ & $\begin{array}{l}\text { I.3.EFINT1. } \mathrm{N}^{\circ} \text { de empresas que lograron realizar actividades } \\
\text { internacionales ((período fijado: p.e., dentro del año posterior a la } \\
\text { asistencia recibida). } \\
\text { I.3.EFINT.2. } \mathrm{N}^{\circ} \text { de empresas que lograron incrementar activida- } \\
\text { des internacionales ((período fijado: p.e., dentro del año posterior } \\
\text { a la asistencia recibida). }\end{array}$ & & & & & \\
\hline
\end{tabular}

FUENTE: Elaboración propia. 


\section{Tabla 7. Cuadro de Indicadores para la función de Modernización Tecnológica, según procesos del Mapa Estratégico}

\begin{tabular}{|c|c|c|c|c|c|c|}
\hline $\begin{array}{l}\text { FUNCIÓN A } \\
\text { EVALUAR }\end{array}$ & INDICADORES & $\begin{array}{l}\text { UNIDAD/ } \\
\text { PUESTO } \\
\text { RESPON- } \\
\text { SABLE } \\
\text { MEDICIÓN }\end{array}$ & $\begin{array}{l}\text { UNIDAD/ } \\
\text { PUESTO } \\
\text { RESPON- } \\
\text { SABLE } \\
\text { GESTIÓN }\end{array}$ & $\begin{array}{l}\text { PLA- } \\
\text { ZO O } \\
\text { PERÍO- } \\
\text { DO }\end{array}$ & $\begin{array}{l}\text { MEDIDA } \\
\text { ESTÁN- } \\
\text { DAR O } \\
\text { DESEADA }\end{array}$ & $\begin{array}{c}\text { DESVIA- } \\
\text { CIÓN EN } \\
\text { MEDIDA } \\
\text { REAL }\end{array}$ \\
\hline & $\begin{array}{l}\text { 4. MODERNIZACIÓN TECNOLÓGICA (especial atención a } \\
\text { Alfabetización Digital y Digitalización Progresiva, UE) }\end{array}$ & & & & & \\
\hline $\begin{array}{c}\text { APTEC. } \\
\text { INICIATIVAS } \\
\text { APOYO } \\
\text { MODER- } \\
\text { NIZACIÓN } \\
\text { TECNOLÓ- } \\
\text { GICA } \\
\text { EMPRESAS } \\
\text { LOCALES }\end{array}$ & $\begin{array}{l}\text { S.4.APTEC.1. No de iniciativas locales para apoyo en la moder- } \\
\text { nización tecnológica } \\
\text { S.4.APTEC.2. Variación anual del } n^{\circ} \text { de iniciativas locales para } \\
\text { apoyo a empresas } \\
\text { E.4.APTEC.1. Ingresos/Costes de actividad apoyo } \\
\text { E.4APTEC.2. Variación anual del ratio Ingreso/Costes Apoyo } \\
\text { P.4.APTEC.1. Presupuesto destinado a iniciativas locales apoyo } \\
\text { P.4.APTEC.2. Variación presupuestaria anual Iniciativas locales } \\
\text { apoyo } \\
\text { I.4.APTEC.1. Grado de satisfacción de las empresas asociadas } \\
\text { con esta labor }\end{array}$ & & & & & \\
\hline $\begin{array}{c}\text { FTEC. } \\
\text { FORMACIÓN } \\
\text { PARA LA } \\
\text { MODER- } \\
\text { NIZACIÓN } \\
\text { TECNOLÓ- } \\
\text { GICA }\end{array}$ & $\begin{array}{l}\text { I.4.FTEC.1. } N^{\circ} \text { de actividades para diagnosis necesidades } \\
\text { formativas empresas } \\
\text { I.4.FTEC.2. Variación anual (u otro período) } n^{\circ} \text { actividades } \\
\text { diagnosis necesidades formativas } \\
\text { S.4.FTEC.1. } N^{\circ} \text { de actividades formativas } \\
\text { S.4.FTEC.2. Variación anual del } n^{\circ} \text { de actividades formativas } \\
\text { S.4.FTEC.3. } N^{\circ} \text { de participantes formandos } \\
\text { S.4.FTEC.4. Variación anual del } n^{\circ} \text { de formandos } \\
\text { S.4.FTEC.5. N }{ }^{\circ} \text { de horas de formación } \\
\text { S.4.FTEC.6. Variación anual de horas formación } \\
\text { S.4.FTEC.7. Contenidos priorizados en formación } \\
\text { I.4.FTEC.3. Grado de satisfacción de formandos y empresas }\end{array}$ & & & & & \\
\hline $\begin{array}{l}\text { ICTEC. } \\
\text { INGRESOS Y } \\
\text { COSTES DE } \\
\text { MODER- } \\
\text { NIZACIÓN } \\
\text { TECNOLÓ- } \\
\text { GICA }\end{array}$ & $\begin{array}{l}\text { E.4.ICTEC.1. Ingresos/Costes actividades Modernización } \\
\text { Tecnológica (general) } \\
\text { E.4.ICTEC.2. Ingresos/Costes actividades Alfabetización Digital } \\
\text { E.4.ICTEC.3. Ingresos/Costes actividades avance en Digitali- } \\
\text { zación } \\
\text { E.4.ICTEC.4. Variación anual de estos ratios } \\
\text { P.4.ICTEC.1. Presupuesto destinado a actividades de Moderni- } \\
\text { zación Tecnológica (general) } \\
\text { P.4.ICTEC.2. Presupuesto destinado a actividades de Alfabeti- } \\
\text { zación Digital } \\
\text { P.4ICTEC.3. Presupuesto destinado a actividades avance en } \\
\text { Digitalización } \\
\text { P.4ICTEC.4. Variación presupuestaria anual para estas activi- } \\
\text { dades }\end{array}$ & & & & & \\
\hline $\begin{array}{l}\text { EFTEC. EFI- } \\
\text { CIENCIA EN } \\
\text { MODER- } \\
\text { NIZACIÓN } \\
\text { TECNOLÓ- } \\
\text { GICA }\end{array}$ & $\begin{array}{l}\text { I.4. EFTEC.1. No de empresas que lograron realizar actividades } \\
\text { de modernización tecnológica (período fijado: p.e., dentro del } \\
\text { año posterior a la asistencia recibida). } \\
\text { I.4.EFTEC.2. } N^{\circ} \text { de empresas que lograron alfabetizarse o pro- } \\
\text { gresar digitalmente ((período fijado: p.e., dentro del año posterior } \\
\text { a la asistencia recibida). }\end{array}$ & & & & & \\
\hline
\end{tabular}

FUENTE: Elaboración propia. 


\section{Tabla 8. Cuadro de Indicadores para la función de Información y Representación, según procesos del Mapa Estratégico}

\begin{tabular}{|c|c|c|c|c|c|c|}
\hline $\begin{array}{l}\text { FUNCIÓNA } \\
\text { EVALUAR }\end{array}$ & INDICADORES & $\begin{array}{l}\text { UNIDAD/ } \\
\text { PUESTO } \\
\text { RESPON- } \\
\text { SABLE } \\
\text { MEDICIÓN }\end{array}$ & $\begin{array}{l}\text { UNIDAD/ } \\
\text { PUESTO } \\
\text { RESPON- } \\
\text { SABLE } \\
\text { GESTIÓN }\end{array}$ & $\begin{array}{l}\text { PLA- } \\
\text { ZO O } \\
\text { PERÍO- } \\
\text { DO }\end{array}$ & $\begin{array}{l}\text { MEDIDA } \\
\text { ESTÁN- } \\
\text { DAR O } \\
\text { DESEADA }\end{array}$ & $\begin{array}{c}\text { DESVIA- } \\
\text { CIÓN EN } \\
\text { MEDIDA } \\
\text { REAL }\end{array}$ \\
\hline & 5. INFORMACIÓN Y REPRESENTACIÓN & & & & & \\
\hline $\begin{array}{c}\text { INFR. } \\
\text { INFRAES- } \\
\text { TRUCTURAS }\end{array}$ & $\begin{array}{l}\text { I.5.INFR.1. No de órganos o instituciones en los que se participa } \\
\text { junto a Administraciones Públicas para asuntos de infraestruc- } \\
\text { turas } \\
\text { I.5.INFR.2. Variación anual de este parámetro } \\
\text { I.5.INFR.3. No de programas o proyectos en los que se participa } \\
\text { junto a Administraciones Públicas para asuntos de infraestruc- } \\
\text { turas } \\
\text { I.5.INFR.4. Variación anual de este parámetro } \\
\text { I.5.INFR.5. No de convenios firmados en los que se participa jun- } \\
\text { to a Administraciones Públicas para asuntos de infraestructuras } \\
\text { I.5.INFRR.6. Variación anual de este parámetro } \\
\text { S.5.INFR.1. No de actividades realizadas en desarrollo de esta } \\
\text { participación } \\
\text { S.5.INFR.2. Variación anual de este parámetro } \\
\text { I.5.INFR.7. Grado de satisfacción de las empresas asociadas } \\
\text { con esta labor }\end{array}$ & & & & & \\
\hline $\begin{array}{c}\text { POL. } \\
\text { POLÍTICA } \\
\text { ECONÓMI- } \\
\text { CO-EMPRE- } \\
\text { SARIAL }\end{array}$ & $\begin{array}{l}\text { 1.5.POL.1. No de órganos o instituciones en los que se participa } \\
\text { junto a Administraciones Públicas para asuntos de política } \\
\text { económica-empresarial } \\
\text { I.5.POL.2. Variación anual de este parámetro } \\
\text { I.5.POL.3. No de programas o proyectos junto a Administraciones } \\
\text { Públicas para asuntos de política económica-empresarial } \\
\text { I.5.POL.4. Variación anual de este parámetro } \\
\text { I.5.POL.5. No de convenios firmados en los que se participa } \\
\text { junto a Administraciones Públicas para asuntos de política } \\
\text { económica-empresarial } \\
\text { I.5.POL.6. Variación anual de este parámetro } \\
\text { S.5.POL.1. No de actividades realizadas en desarrollo de esta } \\
\text { participación } \\
\text { S.5.POL.2. Variación anual de este parámetro } \\
\text { I.5.POL.7. Grado de satisfacción de las empresas asociadas } \\
\text { con esta labor }\end{array}$ & & & & & \\
\hline $\begin{array}{c}\text { ENT. } \\
\text { ENTORNO Y } \\
\text { ESTRATEGIA }\end{array}$ & $\begin{array}{l}\text { I.5.ENT.1. No de proyectos en los que se participa para la inves- } \\
\text { tigación observación y recopilación de información estratégica } \\
\text { sobre el entorno empresarial } \\
\text { I.5.ENT.2. Variación anual de este parámetro } \\
\text { S.5.ENT.1. No de actividades realizadas en desarrollo de esta } \\
\text { participación } \\
\text { S.5.ENT.2. Variación anual de este parámetro } \\
\text { I.5.ENT.3. Grado de satisfacción de las empresas asociadas con } \\
\text { esta labor. }\end{array}$ & & & & & \\
\hline
\end{tabular}




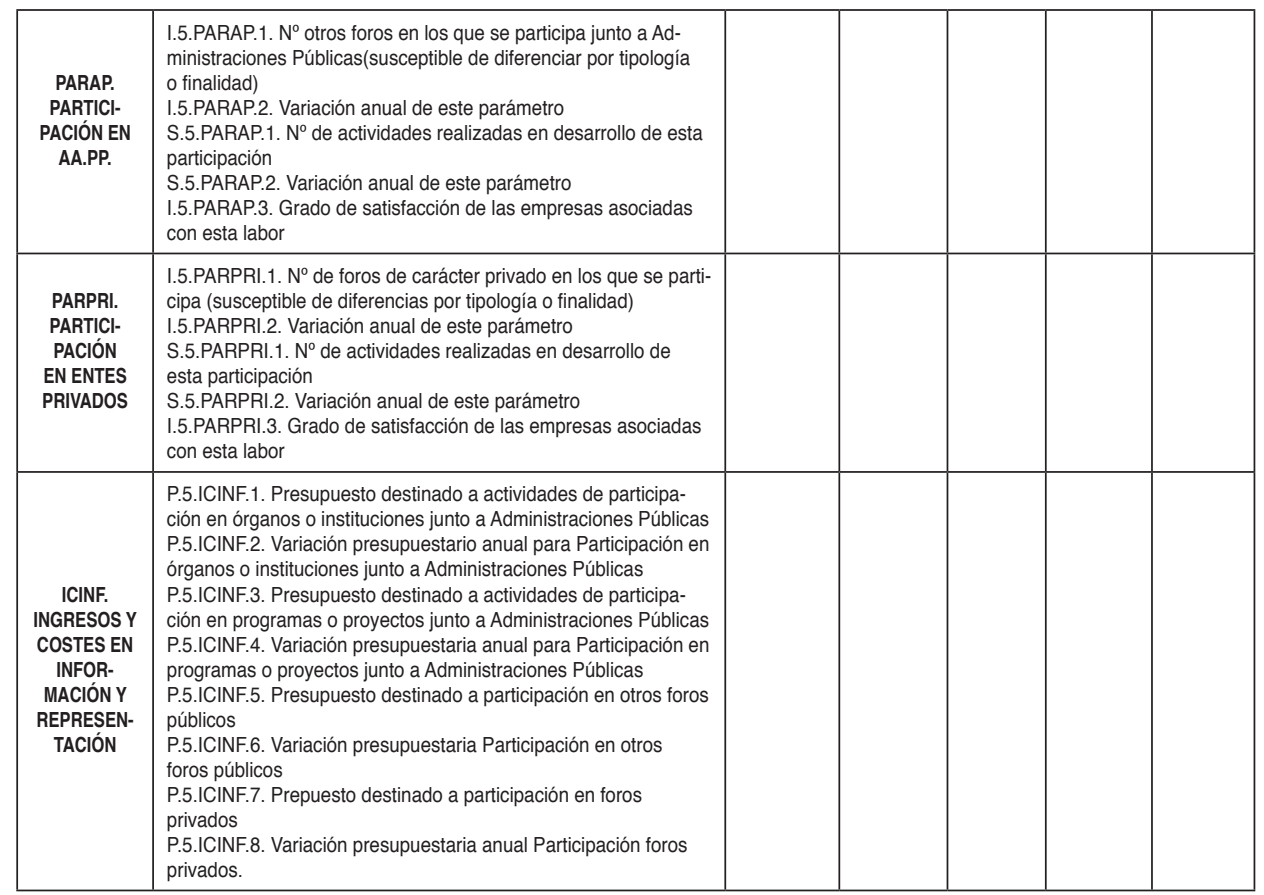

FUENTE: Elaboración propia.

\section{Referencias bibliográficas}

ABDULLAH, I., UMAIR, T., RASHID, Y. \& NAEEM, B. (2013): "Developments on Balanced Scorecard: A Historical Review", World Applied Sciences Journal, 21(1), 134 141. DOl:10.5829/idosi. wasj.2013.21.1.2314.

AL-HAYALY, M.A.M. \& ALNAJJAR, F.J.S. (2016): "Knowledge Management Processes and Their Impact on Organizational Performance, the Adoption Balanced Scorecard: The Moderating Role of Quality Assurance Standards - An Applied Study on Private Jordanian Universities", International Journal of Business and Management, 11(6), 70. DOI:10.5539/ijbm.v11n6p70. 
ALZATE CARDONA, P.F. (2007): "Gestión de los objetivos estratégicos en el sector solidario de Caldas soportado en el Cuadro de Mando Integral (cooperativas de ahorro y crédito, cooperativas de aportes y crédito y fondos de empleados)", Tesis para optar el título de Magíster en Administración, Universidad Nacional de Colombia, Facultad de Ciencias y Administración, Manizales. https:// repositorio.unal.edu.co/handle/unal/2732.

APARISI, J.A., GINER, A. \& RIPOLL, V. (2009): "Análisis del proceso de implantación de un sistema de gestión estratégica. estudio de caso del Cuadro de Mando Integral en la Autoridad Portuaria de Valencia", Revista Española de Contabilidad y Financiación, 38 (142), 189-212. DOI:10.1080/ 02102412.2009.10779666.

ARGÜELLO, E. \& QUESADA, C. (2015): "Implementación del Cuadro de Mando Integral en Pequeñas Empresas: una revisión de literatura", Ciencias Económicas, 33(2), 80, DOI:10.15517/ rce.v33i2.22227.

ASOCIACIÓN ESPAÑOLA DE CONTABILIDAD Y ADMINISTRACIÓN DE EMPRESAS, AECA (2006): "La Contabilidad de Gestión en el Sistema Portuario Español", Principios de Contabilidad de Gestión, 31.

ASOCIACIÓN ESPAÑOLADE CONTABILIDAD Y ADMINISTRACIÓN DE EMPRESAS, AECA (2002): "Indicadores para la Gestión Empresarial", Principios de Contabilidad de Gestión, 17.

ASOCIACIÓN ESPAÑOLA DE CONTABILIDAD Y ADMINISTRACIÓN DE EMPRESAS, AECA (2001): "Gestión Estratégica de Costes", Principios de Contabilidad de Gestión, 23,

ASSIRI, A., ZAIRI, M. \& EID, R. (2006): "How to Profit from the Balanced Scorecard: An Implementation Roadmap", Industrial Management \& Data Systems, 106, 7:937-952. DOI: $10.1108 / 026355706106888669$.

AWADALLAH, E.A. \& ALLAM, A. (2015): "A Critique of the Balanced Scorecard as a Performance Measurement Tool", International Journal of Business and Social Science, 6 (7), 91-99. http:// ijbssnet.com/journals/Vol_6_No_7_July_2015/9.pdf.

BASUONY, M.A.K. (2014): "The Balanced Scorecard in large firms and SMEs: A critique of the nature, value and application", Accounting and Finance Research, 3 (2), 14-22, DOI: 10.5430/afr.v3n2p14.

BERNHARD SMANDEK, A.B., WINKLER, J. \& ULBIG, P. (2010): "Balanced score card implementation for IP rights management in a public research institution", Measuring Business Excellence, 14, 4, 65-75. DOI:10.1108/13683041011093767.

BINDEN, W., MZIU, H. \& SUHAIMI, M.A. (2014): "Employing the Balanced Scorecard (BSC) to Measure Performance in Higher Education-Malaysia", International Journal of Information and Communication Technology Research, 4(1), 38-44, https://pdfs.semanticscholar. org/14d8/98c29ad8f5890e32a9aada739900018202fa.pdf. 
BLANCO CONSTANS, F. (1945): "Estudios Elementales de Derecho Mercantil", tomo II, citado en voz Cámaras de Comercio, Industria y Navegación, Enciclopedia Jurídica Española, Tomo IV, 804 y ss.

BRIONES PEÑALVER, A.J. (2015): Análisis y planificación estratégica de la Cofradía de Pescadores de Cartagena, Escuela Politécnica de Cartagena, Facultad de Ciencias de la Empresa.

CABRA DE LUNA, M.A. (2001): "Propuesta de balance social para fundaciones", CIRIEC-España, Revista de Economía Pública, Social y Cooperativa, 39, 51-78. http://ciriec-revistaeconomia.es/ wp-content/uploads/03_Cabra_de_Luna_39.pdf.

CHAVAN, M. (2009): "The balanced scorecard: a new challenge", Journal of Management Development, 28, 5, 393-406, DOI: 10.1108/02621710910955930.

COKINS, G. (2010): "The promise and perils of the balanced scorecard", Journal of Corporate Accounting \& Finance, 21(3), 19-28. DOI: 10.1002/jcaf.20576.

DANIEL, E., MYERS, A. \& DIXON, K. (2008): "Don't Blame the Tools: The Adoption and Implementation of Managerial Innovations", Research Executive Summaries Series, 4:1-12. https://www. researchgate.net/publication/42797881_Don\%27t_Blame_the_Tools_The_Adoption_and_ Implementation_of_Managerial_Innovations.

DAVID DE SAID, A.C. y LASTRA LUSCALDO, A.L. (2007): Diseño de un sistema de indicadores de gestión bajo las perspectivas del "Balanced Scorecard (BSC) en la Cámara de Comercio de Santa Marta, Fundación Universidad del Norte. División de Ciencias Administrativas. Maestría en Administración de Empresas. Trabajo Grado Magister en Administración de Empresas. Barranquilla, Colombia. http://manglar.uninorte.edu.co/bitstream/handle/10584/159/36536361. pdf?sequence=1\&isAllowed=y.

DE WAAL, A. (2003): "The Future of the Balanced Scorecard: An Interview with Professor Dr Robert S. Kaplan", Measuring Business Excellence. DOI: 10.1108/13683040310466708.

DUVAUCHELLE CALDERON, B.A. \& MERCHÁN LORENTY, A.A. (2019): Diseño de un Cuadro de Mando Integral como modelo de gestión para la Cooperativa de Ahorro y Crédito Universidad de Guayaquil, Universidad de Guayaquil, Facultad de Ciencias Administrativas, Guayaquil, http:// repositorio.ug.edu.ec/handle/redug/38731.

ELOLA, L.N., TEJEDOR, J.P. \& TEJEDOR, A.C.P. (2016): "Analysis of the causal relationships in the balanced scorecard in public and private Spanish Universities through structural equation modelling", The Business \& Management Review, 7(5), 18. https://www.researchgate.net/ publication/319619828_Analysis_of_the_causal_relationships_in_the_balanced_scorecard_in_ public_and_private_Spanish_Universities_through_structural_equation_modelling. 
ESTRADA LLAQUET, J.L. (2007): Mejora de la competitividad de un puerto por medio de un nuevo modelo de gestión de la estrategia aplicando el Cuadro de Mando Integral, Tesis Doctoral, Universidad Politécnica de Madrid, E.T.S. de Ingenieros de Caminos, Canales y Puertos, http:/l oa.upm.es/535/1/JOSE_LUIS_ESTRADA_LLAQUET.pdf.

FIJAŁKOWSKA, J. \& OLIVEIRA, C. (2018): "Balanced Scorecard in Universities", Journal of Intercultural Management, 4:57-83. DOI: 10.2478/joim-2018-0025.

FONCUBIERTA RODRÍGUEZ, M.J. (2012): "Eficiencia empresarial y responsabilidad social en la administración portuaria estatal: el caso de la Autoridad Portuaria de la Bahía de Algeciras", Revista de Responsabilidad Social de la Empresa, 10, 105-134. https://www.accioncontraelhambre.org/ sites/default/files/documents/10_2.pdf.

GARCÍA-LORENZO, I. \& VARELA-LAFUENTE, M.M. (2019): "Interacciones de Gobernanza en las Pesquerías a Pequeña Escala de Galicia: percepciones y dinámicas en las Cofradías de Pescadores", Estudios de Economía Aplicada, 37(3), 42-59. DOI: 10.25115/eea.v37i3.2772.

GONZÁLEZ QUINTANA, M.J. y CAÑADAS MOLINA, E. (2008): "Los indicadores de gestión y el CMI en las ENL", CIRIEC, Revista de economía pública, social y cooperativa, 63, 227-252. http:// ciriec-revistaeconomia.es/wp-content/uploads/6309_Gonzalez_y_Canadas.pdf.

GONZÁLEZ SÁNCHEZ, M.B., LÓPEZ-VALEIRAS SAMPEDRO, E. y GÓMEZ CONDE, J. (2011): "EI Cuadro de Mando Integral en el sector sanitario español: una revisión analítica de la literatura", RIGC, IX, 17, enero-junio. http://www.observatorio-iberoamericano.org/Revista_lberoamericana_ de_Contab_Gesti\%C3\%B3n/N\%C2\%BA\%2017/M_Beatriz_Gonzalez_Ernesto_L\%C3\%B3pezValeiras_Jacobo_G\%C3\%B3mez_Conde.pdf.

GONZÁLEZ SÁNCHEZ, M. \& RÚA ALONSO DE CORRALES, E. (2007): "Análisis de la eficiencia en la gestión de las fundaciones: una propuesta metodológica", CIRIEC-España, Revista de Economía Pública, Social y Cooperativa, 57, 117-149, http://ciriec-revistaeconomia.es/wpcontent/uploads/5705_Gonzalez_y_Rua.pdf.

GORORDO BILBAO, J.M. (2005): Cámaras Oficiales de Comercio, Industria y Navegación, Cizur Menor (Navarra): Ed. Aranzadi.

GUIMARÃES, B., SIMÕES, P. \& MARQUES, R.C. (2010): "Does Performance Evaluation Help Public Managers? A Balanced Scorecard Approach in Urban Waste Services", Journal of Environmental Management, 91, 2632-2638, DOI: 10.1016/j.jenvman.2010.07.039.

HASANAH, N. \& NOVITA, N. (2019): "Village Fund: a Balanced Scorecard Approach. Accruals", Accounting Research Journal of Sutaatmadja, 3(1), 106-130. DOI: 10.35310/accruals.v3i1.44.

HOQUE, Z. (2014): "20 years of studies on the balanced scorecard: Trends, accomplishments, gaps and opportunities for future research", The British Accounting Review, 46(1), 33-59. DOI: 10.1016/j.bar.2013.10.003. 
HRISTOV, I. \& CHIRICO, A. (2016): "The Limits of the Balanced Scorecard", Open Journal of Social Sciences, 4, 53-58. DOI: 10.4236/jss.2016.411004.

HUANG, H.C., LAI, M.C. \& LIN, L.H. (2011): "Developing strategic measurement and improvement for the biopharmaceutical firm: using the BSC hierarchy", Expert Systems with Applications, 38, 5, 4875-4881. DOI: 10.1016/j.eswa.2010.09.069.

JIANG, D.K. \& LIU, Z.K. (2014): "Research on Application of Balanced Scorecard in the Government Performance Appraisal", Open Journal of Social Sciences, 2, 91-96. DOI: 10.4236/jss.2014.29016.

JUNTA DE ANDALUCÍA (2004): Cuadro de Mando Integral para los Ayuntamientos de menos de 50.000 habitantes, Consejería de Gobernación, Dirección General de Administración Local. https://www.juntadeandalucia.es/organismos/turismoregeneracionjusticiayadministracionlocal/ areas/administracion-local/investigaciones-estudios-admonlocal/paginas/cuadro-mando-integral. $\mathrm{html}$

KAPLAN, R.S., NORTON, D.P. \& RUGELSJOEN, B. (2010): "Managing alliances with the balanced scorecard", Harvard Business Review, 88(1), 114-120. https://www.researchgate.net/ publication/293357033_Managing_Alliances_with_the_Balanced_Scorecard.

KAPLAN, R.S. \& NORTON, D.P. (2008): Execution Premium: Linking Strategy to Operations for Competitive Advantage, Barcelona: Harvard Business Press.

KAPLAN, R.S. \& NORTON, D.P. (2000): "Having Trouble with Your Strategy? Then Map It", Harvard Business Review, september-october, 167-176. https://hbr.org/2000/09/having-trouble-with-yourstrategy-then-map-it.

KAPLAN, R.S. \& NORTON, D.P. (1996): The Balanced Scorecard Translating Strategy into Action, Boston, Massachusetts, USA: Harvard Business School Press.

KAPLAN, R.S. \& NORTON, D.P. (1992): "The Balanced Scorecard: Measures that Drive Performance", Harvard Business Review, january-february, 71-79. https://hbr.org/1992/01/the-balancedscorecard-measures-that-drive-performance-2.

KHIEW, K.-F., CHEN, M.-C., SHIA, B.-C. \& PAN, C.H. (2017): "Adapting the Balanced Scorecard into the HealthCare Industry: A Literature Review, New Insight and Future Directions", Open Journal of Business and Management, 5, 611-623. DOI: 10.4236/ojbm.2017.54052.

KUCHTA, D., RYNCA, R., SKORUPKA, D. \& DUCHACZEK, A. (2019): "The use of the multiple knapsack problem in strategic management of a private Polish university Case study", International Journal of Educational Management, 33, 2, 335-358. DOI: 10.1108/JJEM-03-2017-0068.

LIZCANO ÁLVAREZ, J. (2012): "Un sistema de indicadores de gestión para los Ayuntamientos", Documentos AECA, Principios de Contabilidad de Gestión, Madrid. 
LÓPEZ HERNÁNDEZ, A.M. (2010): Aplicación del Balance Scorecard a los servicios públicos locales deportivos. El caso del Patronato Municipal de Deportes de Granada, Universidad de Granada. http://tecnicasdecep.pbworks.com/f/BALANCE+SCORECARD+PMD+GRANADA.pdf.

LÓPEZ HERNÁNDEZ, A.M., LÓPEZ VILLEGLA, A. \& RODRíGUEZ NIETO, A. (2008): El Cuadro de Mando Integral como herramienta de gestión estratégica en entidades deportivas públicas y privadas, Instituto Andaluz del Deporte. https://www.juntadeandalucia.es/export/drupaljda/ publicacion/20/01/El_cuadro_de_mando_integral.pdf.

LÓPEZ VIÑEGLA, A. (2003): Gestión estratégica y medición. El cuadro de mando como complemento del Balanced Scorecard, Monografía AECA.

LÓPEZ VIÑEGLA A. (2004): Los Sistemas de Información basados en la Estrategia, Ediciones Deusto Planeta de Agostini Profesional y Formación SL. https://buscadorinfo.unan.edu.ni/Record/ ELB14850/Details.

LU, H.Y. (2009): "Local Government Performance Evaluation: Experience, Plight and the Way Out", Chinese Public Administration, 6, 31-33. http://www.cnki.com.cn/Article/CJFDTotalZXGL200906011.htm.

MADSEN, D.O. \& STENHEIM, T. (2015): "The Balanced Scorecard: A Review of Five Research Areas", American Journal of Management, 15(2), 24-41. https://pdfs.semanticscholar.org/60a5/ d36377c38d3031ebe2daa7bad552223ec64c.pdf?_ga=2.240873733.747134890.1590517109885068328.1590517109.

MADSEN, D. O.\& STENHEIM, T. (2014): "Perceived benefits of balanced scorecard implementation: Some preliminary evidence", Problems and Perspectives in Management, 12 (3), 81-90. https:// core.ac.uk/download/pdf/52119771.pdf.

MARÍN, J. \& GARCÍA, O. (2007): "Indicadores para la gestión de las entidades deportivas", Atlas Sport Consulting, $2^{\circ}$ Jornadas, ACEDYR. http://www.atlas-sport.com/_pdf/345528281090705.pdf.

MARTÍNEZ-VILANOVA MARTÍNEZ, A.M. \& RODENES-ADAM, M. (2009): "Sistema de diagnóstico de gestión adaptado del cuadro de mando integral y del modelo EFQM de excelenciaß. Aplicación a las Cajas Rurales", Estudios de Economía Aplicada, 27 (3), 1-30. https://www.researchgate.net/ publication/44389142_Sistema_de_diagnostico_de_gestion_adaptado_del_cuadro_de_mando_ integral_y_del_modelo_EFQM_de_excelenciaR_Aplicacion_a_las_Cajas_Rurales.

MINTZBERG, H. (1994): The Rise and Fall of Strategic Planning, New York, USA: Prentice Hall.

MIRANDA PUREZA, J. \& DALLA VALENTINA, L.V.O. (2006): "Balanced Scorecard: a new approach", Third International Conference on Production Research Americas' Region 2006 (ICPR-AM06).

NAVARRO GALERA, A. (2008): Indicadores y coste de los servicios deportivos de la Administración Pública, Instituto Andaluz del Deporte, Curso sobre Gestión económica del Deporte, Loja (Granada). 
NIVEN, P.R. (2003): El Cuadro de Mando Integral paso a paso, Barcelona: Ediciones Gestión 2000.

NØRREKLIT, H. \& MITCHELL, F. (2014): "Contemporary issues on the balance scorecard", Journal of Accounting \& Organizational Change, 10(4). DOl:10.1108/JAOC-04-2014-0026.

OSPINA, S. (2000): Evaluación de la gestión pública: conceptos y aplicaciones en el caso latinoamericano. $V$ Congreso Internacional del Centro Latinoamericano de Administración para el Desarrollo (CLAD). http://old.clad.org/portal/publicaciones-del-clad/revista-clad-reformademocracia/articulos/019-febrero-2001-1/evaluacion-de-la-gestion-publica-conceptos-yaplicaciones-en-el-caso-latinoamericano-1/view.

PARIONA C.C. \& PEÑA H.H. (2020): "Cuadro de mando integral en la toma de decisiones de las cooperativas agrarias de la región Junín", Prospectiva Universitaria, 12(1-2), 75-80. http://revistas. uncp.edu.pe/index.php/prospectiva/article/view/458/542.

PORTACIO-RODRÍGUEZ, C.A. (2017): "Perspectivas del cuadro de mando integral en cooperativas de transporte Carlos Alberto", FIPCAEC, 4 (2), 49-64. DOI: 10.23857/fipcaec.v2i4.31.

QUESADO, P., AIBAR GUZMÁN, B. \& LIMA RODRÍGUES, L. (2018): "Advantages and contributions in the balanced scorecard implementation", Intangible Capital, 14(1), 186-201. DOI: 10.3926/ic.1110.

RAMÍREZ, Y. (2011): "New management in Spanish universities: introducing Balanced Scorecard", International Journal of Learning and Intellectual Capital, 8 (4). DOI: 10.1504/IJLIC.2011.043061.

REABUÑAY, N.F. (2018): Diseño de un modelo de gestión basado en el Cuadro de Mando Integral para el Sindicato de Chóferes Profesionales del Cantón Colta, Provincia de Chimborazo, Escuela Superior Politécnica de Chimborazo, Facultad de Administración de Empresas, Escuela de Contabilidad y Auditoría, Riobamba-Ecuador. http://dspace.espoch.edu.ec/handle/123456789/9947.

RONCANCIO, G. (2018): Pensemos, Fecha de publicación: 13 de abril de 2018.

RIBEIRO, M.L., VASCONCELOS, M.L. \& ROCHA, F. (2019): "Monitoring performance indicators in the Portuguese hospitality sector", International Journal of Contemporary Hospitality Management, 31, 2, 790-811. DOI: 10.1108/JJCHM-03-2017-0178.

SANTOS, M. \& FIDALGO, E. (2005): "El Balanced Scorecard o Cuadro de Mando Integral y el Cuadro de Mando Tradicional: principales diferencias", Técnica Contable, LVII, 673, Marzo, 13-17. http:// jggomez.eu/z\%20Privado/b\%20usuarios/n-revista/caja/3tc/2005/673.pdf.

SERNA GÓMEZ, H. (2003): Gerencia estratégica, Bogotá: Editorial 3R Panamericana.

VALDÉS PÉREZ, C.A. (2017): Diseño de un Cuadro de Mando Integral para la administración del Colegio de Ingenieros de Chile, Tesis para optar al Grado de Magíster en Gestión y Políticas Públicas, Santiago de Chile. http://repositorio.uchile.cl/handle/2250/146527. 
VEGA FALCÓN, V. \& LLUGLLA JÁCOME, K. (2019): "El Balanced Scorecard como herramienta de gestión organizacional", Ecociencia, 6(2), 1-24, http://revistas.ecotec.edu.ec/index.php/ ecociencia/article/view/187

VEGA, V. (2015): El Cuadro de Mando Integral percibido a través de casos reales, I ed., Quito: Mendieta.

VIÑAS, J. (2009): "EI Cuadro de Mando Integral y su implementación en una organización Deportiva", Ponencia Asociación Aragonesa de Gestores Deportivos, 23 junio 2009.

WEBSTER, D.W. \& COKINS, G. (2020): "The Strategy Map and Its Balanced Scorecard", Chapter Four. DOI: 10.1002/9781119660187.ch4.

YETANO, A. (2005): "El Cuadro de Mando Integral (Balanced Scorecard) en la Administración Local", Auditoria Pública: Revista de los Órganos Autónomos de Control Externo, 36, 39-46. https://www. researchgate.net/publication/28093792_El_cuadro_de_mando_integralbalance_scorecarden_ la_Administracion_Local.

ZACHOW, M. \& BERTOLINI, G.R.F. (2019): "Use of Balanced Scorecard in Cooperatives", International Journal of Advanced Engineering Research and Science (IJAERS), 6, 160-166, DOI: 10.22161/ ijaers.610.25.

\section{Referencias normativas}

Real Decreto de 9 de abril de 1886

[https://www.boe.es/datos/pdfs/BOE//1886/102/A00109-00110.pdf]

Ley de Bases de 29 de junio de 1911

Real Decreto Ley 13/2010, de 3 de diciembre, de actuaciones en el ámbito fiscal, laboral y liberalizadoras para fomentar la inversión y creación de empleo

[https://www.boe.es/buscar/act.php?id=BOE-A-2010-18651].

Ley de Cámaras de Comercio Españolas, Ley 4/2014, de 1 de abril, Básica de las Cámaras Oficiales de Comercio, Industria, Servicios y Navegación

[https://www.boe.es/buscar/doc.php?id=BOE-A-2014-3520]. 